\title{
Physicochemical property space distribution among human metabolites, drugs and toxins Varun Khanna ${ }^{1}$ and Shoba Ranganathan ${ }^{* 1,2}$
}

\author{
Addresses: ${ }^{1}$ Dept. of Chemistry and Biomolecular Sciences \& ARC Centre of Excellence in Bioinformatics, Macquarie University, Sydney, \\ Australia and ${ }^{2}$ Dept. of Biochemistry, Yong Loo Lin School of Medicine, National University of Singapore, Singapore \\ E-mail: Varun Khanna - vkhanna@cbms.mq.edu.au; Shoba Ranganathan* - shoba.ranganathan@mq.edu.au \\ ${ }^{*}$ Corresponding author
}

from Asia Pacific Bioinformatics Network (APBioNet) Eighth International Conference on Bioinformatics (InCoB2009)

Singapore 7-II September 2009

Published: 3 December 2009

BMC Bioinformatics 2009, I0(SuppI I5):SI0 doi: 10.1I86/I47I-2105-10-SI5-SI0

This article is available from: http://www.biomedcentral.com/I47/-2/05/I0/SI5/SI0

(C) 2009 Khanna and Ranganathan; licensee BioMed Central Ltd.

This is an open access article distributed under the terms of the Creative Commons Attribution License (http://creativecommons.org/licenses/by/2.0), which permits unrestricted use, distribution, and reproduction in any medium, provided the original work is properly cited.

\begin{abstract}
Background: The current approach to screen for drug-like molecules is to sieve for molecules with biochemical properties suitable for desirable pharmacokinetics and reduced toxicity, using predominantly biophysical properties of chemical compounds, based on empirical rules such as Lipinski's "rule of five" (Ro5). For over a decade, Ro5 has been applied to combinatorial compounds, drugs and ligands, in the search for suitable lead compounds. Unfortunately, till date, a clear distinction between drugs and non-drugs has not been achieved. The current trend is to seek out drugs which show metabolite-likeness. In identifying similar physicochemical characteristics, compounds have usually been clustered based on some characteristic, to reduce the search space presented by large molecular datasets. This paper examines the similarity of current drug molecules with human metabolites and toxins, using a range of computed molecular descriptors as well as the effect of comparison to clustered data compared to searches against complete datasets.

Results: We have carried out statistical and substructure functional group analyses of three datasets, namely human metabolites, drugs and toxin molecules. The distributions of various molecular descriptors were investigated. Our analyses show that, although the three groups are distinct, present-day drugs are closer to toxin molecules than to metabolites. Furthermore, these distributions are quite similar for both clustered data as well as complete or unclustered datasets.

Conclusion: The property space occupied by metabolites is dissimilar to that of drugs or toxin molecules, with current drugs showing greater similarity to toxins than to metabolites. Additionally, empirical rules like Ro5 can be refined to identify drugs or drug-like molecules that are clearly distinct from toxic compounds and more metabolite-like. The inclusion of human metabolites in this study provides a deeper insight into metabolite/drug/toxin-like properties and will also prove to be valuable in the prediction or optimization of small molecules as ligands for therapeutic applications.
\end{abstract}




\section{Background}

To search for biologically active compounds, with favorable ADMET [1] (Absorption, Distribution, Metabolism, Excretion, and Toxicity) properties from the immense "chemical space" is a non-trivial task [2]. Druglikeness has been dominated, in the past decade, by Lipinski's "Rule of Five" (Ro5) [3], which states that a compound is likely to be "non-drug-like" if it has more than five hydrogen bond donors, more than 10 hydrogen bond acceptors, molecular mass is greater than 500 and lipophilicity is above 5. The analysis carried out by Leeson and Davis [4] of the approved drugs released before 1983 (i.e. pre-Ro5 era) and the drugs released in between 1983 and 2002 clearly indicates the impact of Ro5 on drug discovery projects.

However, Lipinski's rule has many exceptions and in one of the studies [5] it was shown that using the above criteria, only $66 \%$ of approved drugs in the MDL Drug Data Report (MDDR) database, were classified as druglike; whereas $75 \%$ of the theoretically non-drug-like compounds from the Available Chemical Directory (ACD) were in fact regarded as drug-like by Ro5. Moreover, Ro5 does not select metabolites because metabolite-likeness is a recent measure, since Ro5 was formulated a decade ago, with little knowledge on metabolites and pathways. Similar studies have spurred the quest for new approaches to classify drugs from nondrug molecules $[6,7]$, and to characterize the properties of drug-like or lead-like compounds [8,9]. Subsequently, the "rule-of-three" (Ro3) [10] was proposed for fragment-based lead discovery. Ro3 states that successful hits possess an average Molecular weight $<=300$, the number of hydrogen bond donors $<=3$, the number of hydrogen bond acceptors $<=3$ and $C \log \mathrm{P}<=3$. In addition, the number of rotatable bonds $<=3$ and the polar surface area $<=60$ are also useful in characterizing drug-like and non-drug-like molecules. In past few years, researchers have developed a range of indices, such as the natural product index [11], the metabolite index [12], peptidelikeness [13], lead-likeness [14-16], and drug-likeness [3], in an attempt to achieve a better classification between drugs and non-drugs. In conjunction with machine learning techniques, like Artificial Neural Networks (ANN) [6,7], Support Vector Machine (SVM) [17] and Hidden Markov Models (HMM), statistical [18] and substructure analyses have become widely accepted to characterize the properties of drug-like datasets and reduce the attrition rates in drug development.

\section{Drug-likeness in natural products and synthetic compounds}

In this section, we present a summary of analysis reports primarily focused on identifying drug-likeness in natural products and synthetic organic compounds, derived from combinatorial functional group replacement. Henkel et al. [18] carried out statistical analysis to determine the properties and structural differences between natural products (NPs) and combinatorial molecules. In their analysis, NPs were derived from Chapman and Hall Dictionary of Natural products and the bioactive natural product database (BNPD) obtained from Szenzor Management Consulting Company. These were compared with synthetic compounds from the Available Chemical Directory (ACD) and Bayers database and representative bioactive molecules from drug databases. Stahura et al. [19] used Shannon entropy to analyze the differences between NPs obtained from the Dictionary of Natural Products and synthetic molecules obtained from ACD database. Feher and Schmidt [20] examined representative set of molecules from NPs obtained from four databases namely BioSPECS natural product database, ChemDiv natural product database, Interbioscreeen IBS2001N and HTS-NC database, drugs obtained from (Chapman and Hall Dictionary of Drugs) and combinatorial molecules obtained from (MayBridge HTS database, ChemBridge EXPRESS-Pick database ComGenex Collection, ChemDiv Collection and SPECS screening compound database). The authors concluded that the number of chiral centers, the number of rotatable bonds and the ratio of aromatic atoms to ring atoms are the most distinguishing features among the three classes of compounds. In their study, drugs occupied the property space between NPs and combinatorial compounds, consistent with drugs being obtained from NPs as well as combinatorial libraries. The first three principal components accounted for about $66 \%$ of the variance. Feher and Schmidt were thus the first to introduce the idea of NP-like filters. Lee and Schneider [21] utilized Self Organizing Maps (SOM) for the classification of drugs, non-drugs and NPs. Their study revealed several pharmacophoric patterns in common between NPs and drugs, suggesting the use of such patterns for exploring drug relevant pharmacophoric space.

\section{Metabolite-likeness as the criterion for lead discovery}

With the growing knowledge of biochemical pathways and their cognate metabolites, Hattori et al. [22] analyzed the molecular diversity of KEGG (Kyoto Encyclopedia of Genes and Genomes) Ligand database which includes 9,383 chemical structures. Nobeli et al. [23] have produced an interesting classification of Escherichia coli metabolome according to fragmentbased fingerprints and maximum common subgraphs. Gupta and Aires-de-Sousa [12] compared the structural coverage of the metabolite molecules from the KEGG database and purchasable molecules from the ZINC 
library, a free database of commercially available compounds. They reported the use of various machine learning techniques like Kohonen maps, random forest (RFs) and classification trees to distinguish between metabolites and non-metabolites. Cherkasov [24] and coworkers derived 20 binary classifiers and achieved $99 \%$ of the accurate separation between drugs, drug-like compounds ("druglikes"), bacterial and human metabolites and antimicrobial compounds, and proposed metabolite-likeness as a potential tool for discovering novel antimicrobials. Recently, Dobson et al. [25] compared different molecular properties among human metabolites, drugs and "predrugs" (precursor drug molecules). They concluded that although metabolites are a distinct class of compounds, metabolites and drugs occupy a significant amount of common property space. They further suggested that metabolite-likeness may be used as a filter for designing drugs which are functionally similar to metabolites and thus have better ADMET properties.

The several excellent studies described above have each compared different datasets, using a variety of chemoinformatics tools and molecular descriptors. Furthermore, some of the studies used datasets that were clustered [25], while others have searched or compared complete (unclustered) datasets [24]. Most importantly, the property space of toxic compounds has not been included in any of these studies, whereas one of the basic tenets of drug development to reduce or eliminate toxicity [26].

The analysis carried out of the drug failures during past few decades have shown that over $90 \%$ of the failures are due to high toxicity $[27,28]$. It is therefore essential that the property space of toxins is explored along with drugs and metabolites to develop filters for toxicity.

Our aim is to compare freely available datasets of metabolites, drugs and toxins, as benchmark datasets, using a range of available molecular descriptors, to identify the property space occupied by these three data types. We also present analysis results from complete datasets, as well as clustered datasets, to determine whether clustering molecules would affect the analysis results. Our results indicate that clustering does not affect property distributions to a significant level and that unclustered datasets can be used in drug discovery pipelines. We also report, for the first time to the best of our knowledge, that current drug molecules are more akin to toxins than to metabolites, in physicochemical property space.

\section{Results}

\section{Rule of five (Ro5) analysis}

The number of molecules adhering to Ro5 was calculated and the results are reported in Table 1. It is surprising to note that although Ro5 was formulated to pick out drugs or drug-like molecules, it actually does well in identifying toxin molecules. Over $90 \%$ of the toxin molecules satisfy all Ro5 criteria. On the other hand, metabolites perform worst among the three datasets while drugs do fairly well, as expected due to the predominance of Ro5 over the past decade. It should also be noted that among the four properties compared, the numbers of hydrogen bond donor and acceptor seem to be more robust properties, as over $84 \%$ of the molecules in all the datasets satisfy Ro5 requirements.

\section{Examining the molecular properties of three datasets}

The distribution of various descriptors (properties) among drugs, human metabolites and toxin molecules are available from Table 2 and Fig. 1, 2, 3, 4, based on the analyses of clustered datasets (details in the Methods section). There is very little overlap in the clustered datasets and so no further reduction in redundant data has been carried out (details in the Methods section and Fig. 5).

While there is a multitude of molecular descriptors available for carrying out comparison studies, given the large size of the datasets, we need a set of rapidly computable molecular descriptors, for efficient analysis. Furthermore, to account for $70 \%$ of the drugs, Oprea et al. [9] used simple descriptors such as the count of rings and rotatable bonds along with Lipinski descriptors. We have considered a range of $1 \mathrm{D}$ and $3 \mathrm{D}$ properties for the current analysis. The results are presented as Lipinski (Ro5) properties, 1D properties (non-Ro5 measures) and 3D properties.

Table I: Distribution of molecules following Lipinski's rule

\begin{tabular}{|c|c|c|c|c|}
\hline \multirow[t]{2}{*}{ Datasets } & \multicolumn{4}{|c|}{ Lipinski Properties } \\
\hline & Molecular weight $<500 \mathrm{Da}$ & H-bond Donor <=5 & H-bond Acceptor $<=10$ & $\log P<5$ \\
\hline HMDB (Metabolites) & $34 \%$ & $84 \%$ & $84 \%$ & $35 \%$ \\
\hline DDB (Drugs) & $84 \%$ & $86 \%$ & $87 \%$ & $92 \%$ \\
\hline CPDB (Toxins) & $94 \%$ & $98 \%$ & $97 \%$ & $92 \%$ \\
\hline
\end{tabular}


Table 2: Comparison of molecular properties among the three datasets

\begin{tabular}{|c|c|c|c|}
\hline \multirow[t]{2}{*}{ Molecular Property } & \multicolumn{3}{|c|}{ Mean (Median) \pm std. dev. } \\
\hline & Metabolites & Drugs & Toxins \\
\hline \multicolumn{4}{|l|}{ Lipinski properties } \\
\hline Molecular weight & $621(701) \pm 322$ & $355(309) \pm 259$ & $275(239) \pm 167$ \\
\hline Alog P & $7(10) \pm 7$ & $.08(1) \pm 3.5$ & $2(2) \pm 2$ \\
\hline Lipinski HB acceptors & $9(9) \pm 6$ & $7(6) \pm 7$ & $5(4) \pm 4$ \\
\hline \multicolumn{3}{|l|}{ ID properties } & $2(1) \pm 2$ \\
\hline Number of atoms & $43(5 I) \pm 22$ & $24(2 I) \pm 8$ & $16(14) \pm 11$ \\
\hline Number of carbon atoms & $34(4 I) \pm 18$ & $16(14) \pm 12$ & $12(10) \pm 9$ \\
\hline Number of hydrogen atoms & $60(72) \pm 33$ & $23(19) \pm 18$ & $16(12) \pm 12$ \\
\hline Number of nitrogen atoms & $1(1) \pm 2$ & $3(2) \pm 3$ & $2(1) \pm 2$ \\
\hline Number of oxygen atoms & $8(8) \pm 5$ & $5(4) \pm 5$ & $3(2) \pm 3$ \\
\hline Number of rings & $I(0) \pm 2$ & $3(2) \pm 2$ & $2(2) \pm 2$ \\
\hline Number of ring assemblies & I $(0) \pm 1$ & $2(2) \pm 1$ & $I(1) \pm 1$ \\
\hline Number of rotatable bonds & $27(37) \pm 20$ & 6 (4) \pm 7 & $3(2) \pm 4$ \\
\hline Number of aromatic bonds & I $(0) \pm 4$ & $8(6) \pm 7$ & $6(6) \pm 6$ \\
\hline $\log D$ & $6(9) \pm 7$ & $0.4(0.9) \pm 4$ & $2(1.4) \pm 2.6$ \\
\hline Mol. solubility & $-10(-13) \pm 8$ & $-3(-3) \pm 3$ & $-3(-2) \pm 3$ \\
\hline \multicolumn{4}{|l|}{ 3D properties } \\
\hline Mol. SA & $65 \mid(788) \pm 343$ & $364(316) \pm 252$ & $270(233) \pm 159$ \\
\hline Mol. volume & $450(548) \pm 244$ & $245(214) \pm 170$ & $179(153) \pm 110$ \\
\hline Mol. polar SA & $143(126) \pm 94$ & $121(95) \pm 117$ & $84(63) \pm 76$ \\
\hline Mol. SA volume & $866(105 I) \pm 420$ & $510(464) \pm 272$ & $40 I(366) \pm 164$ \\
\hline Mol. polar sa SA & $216(195) \pm 138$ & $191(156) \pm 173$ & $126(105) \pm 91$ \\
\hline Mol. sa SA & $1034(1205) \pm 472$ & $578(523) \pm 313$ & $451(408) \pm 187$ \\
\hline
\end{tabular}

For each dataset, the mean, median and standard deviation values are provided, with properties ordered as Lipinski properties, ID properties and 3D properties. HB: hydrogen bond; Mol.: Molecular; sa: solvent accessible; SA: Surface Area.

\section{Lipinski properties}

\section{Molecular weight}

Metabolites follow a bimodal distribution in molecular weight, with the first peak at 100-400 (almost 31\% of the dataset) and the second and larger peak at 700-1000, containing $48 \%$ of the dataset. On the other hand, the molecular weight of drugs follows a Gaussian distribution with the majority of drugs $(82 \%)$ under the range of 500. This is in accordance with the Lipinski restriction of weight less than or equal to 500. Despite the Ro5 restriction, $18 \%$ of the drug molecules possess a molecular weight in excess of 500. Toxin molecules more or less follow the same pattern as drugs, with the gradual decrease in number of compounds as molecular weight increases from 100 to 500 (Fig. 1a).

From the calculated mean and median values for the molecular weight, it appears that the metabolite data is skewed towards high molecular weight compounds whereas drugs and toxin molecules prefer a lower molecular weight distribution. The statistics of the molecular weight property for the three datasets are available in Table 2.

Lipophilicity (Alog P)

Lipid solubility is a direct measure of transport abilities of the compound across biological membranes [29].
Drug molecules should have enough solubility to traverse the membrane but should not be too soluble so as to get trapped in them. Thus, lipophilicity of a compound is of special significance in drug discovery programs. The most commonly used parameter to evaluate lipid solubility is the n-octanol/water partition coefficient (Alog P). Positive values of this partition coefficient correspond to a preference for lipophilic or hydrophobic environment while negative values indicate a preference for lipophobic or hydrophilic environment. It is clear from Table 2 and Fig. 1b, that metabolites in general are more lipophilic than drugs or toxic compounds. Only $17 \%$ of the metabolites have negative Alog $P$ values confirming that the majority of the metabolites are lipophilic. On the other hand, 39\% of the drugs have Alog $\mathrm{P}$ values in negative territory, indicating that twofifths of the drugs are lipophobic. Like metabolites, only $19 \%$ of the toxin molecules have negative Alog $\mathrm{P}$ values while the majority of the molecules are in the range 0 to +5 which is much smaller range as compared to metabolites (Fig. 1b).

Lipinski hydrogen bond donors

Lipinski hydrogen bond donors (LHBDs) are determined by counting the numbers of $\mathrm{OH}$ and $\mathrm{NH}$ bonds in each molecule [3]. Approximately 21\% of the metabolites, $12 \%$ of the drugs and $34 \%$ of the toxin molecules do not 

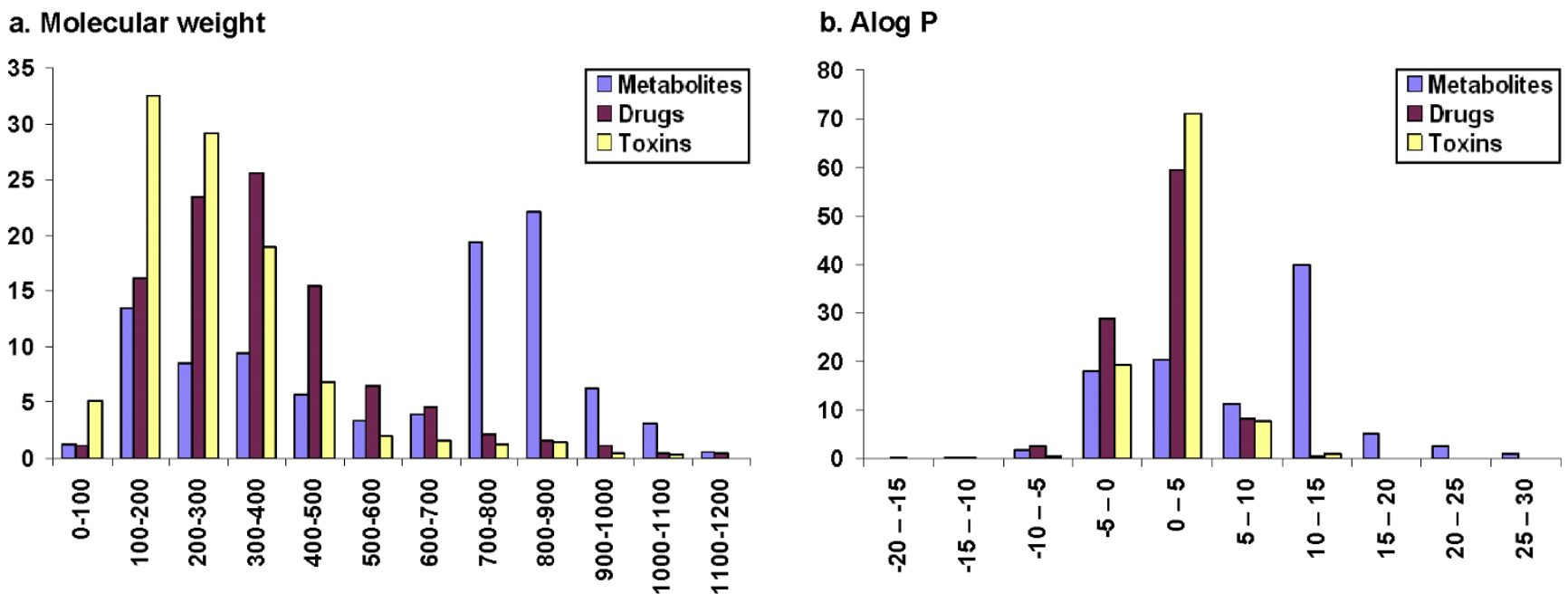

\section{c. Lipinski hydrogen bond donor}

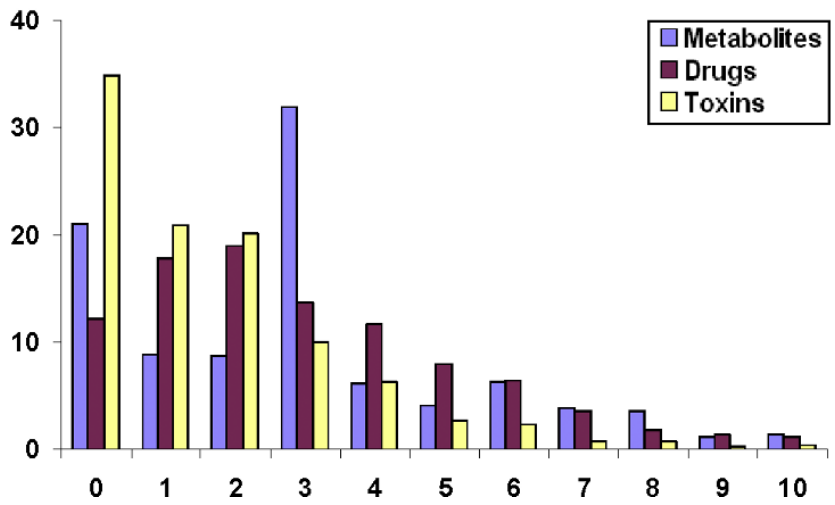

\section{d. Lipinski hydrogen bond acceptor}

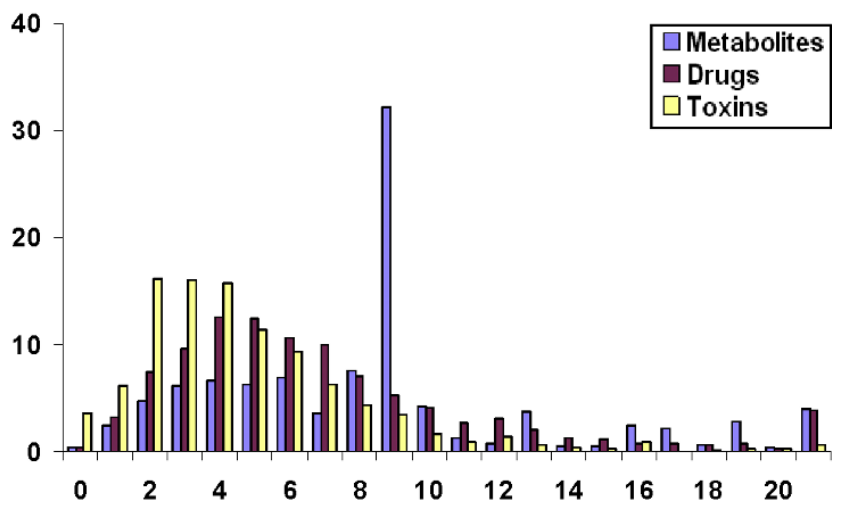

Figure I

Comparison of Lipinski properties among human metabolites, drugs and toxins. Compared properties include

a. Molecular weight, b. AlogP, c. Number of Lipinski hydrogen bond donors and d. Number of Lipinski hydrogen bond acceptors.

possess any LHBDs. Almost the same percentage of molecules in the drug $(\sim 41 \%)$ and toxin $(\sim 36 \%)$ dataset have one or two LHBDs, respectively, while only $17 \%$ of the metabolite dataset has the same number of LHBDs. Only $5 \%$ of the toxins, $14 \%$ of the drugs and $16 \%$ of the metabolites have LHBD greater than five (Fig. 1c).

Lipinski hydrogen bond acceptor

Only a fraction of molecules in all the datasets $(0.35 \%$ of metabolites, $0.40 \%$ of drugs and $3.6 \%$ of toxins) do not possess Lipinski hydrogen bond acceptors (LHBAs), computed by summing the numbers of nitrogen and oxygen atoms in each molecule [3]. Drugs and toxins follow almost the same distribution with the highest percentage of molecules in the range 2-7 LHBA atoms per molecule. On the other hand, metabolites have a wide spread distribution with an unusually high peak at 9 LHBA (Fig. 1d).

\section{ID properties}

Total number of Atoms

The distribution of the total number of atoms in metabolites follows a bimodal pattern (Fig. 2a), with the larger peak at 50-70 atoms, containing 47\% percent molecules and the smaller peak at 10-30 atoms, containing $28 \%$ of molecules. The maximum number of atoms in a metabolite molecule is 124 , while the mean value is 43 atoms per molecule. In contrast to human metabolites, the drug dataset follows a bell-shaped curve, skewed towards low numbers of atoms per molecule. Approximately $79 \%$ of drugs contain 10-40 atoms per molecule. The average number of atoms per molecule in the drug dataset is 24 , while in metabolites, the average is 43. Like drugs, toxin molecules also favor smaller numbers of atoms per molecule, with a mean of 16 and a gradual decrease in the number of compounds as the number of atoms increases per molecule. The majority of 

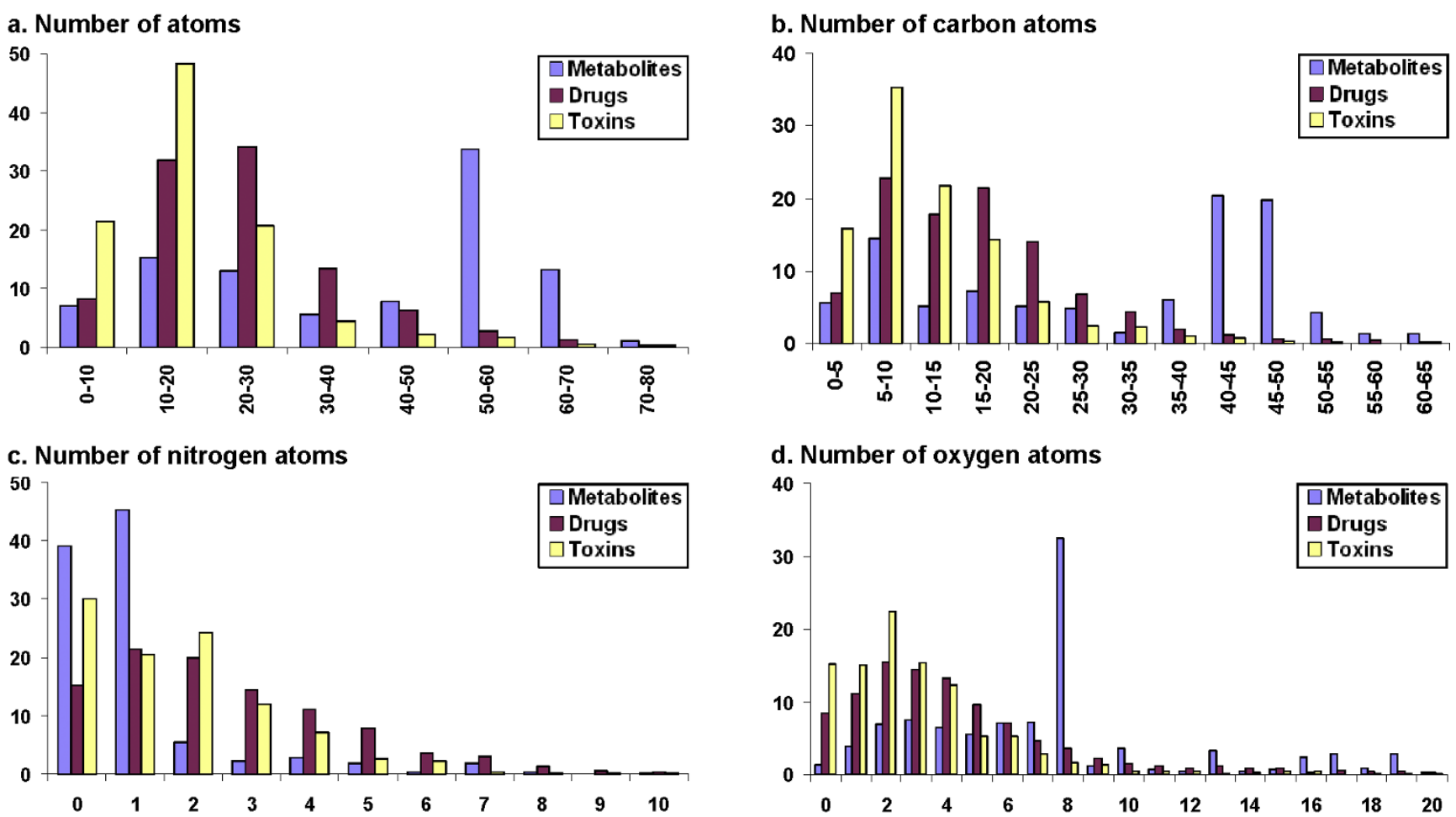

d. Number of oxygen atoms

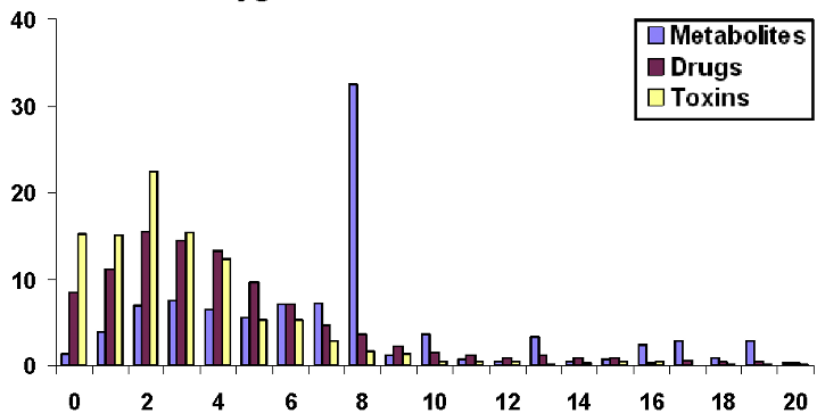

e. Number of positively charged atoms

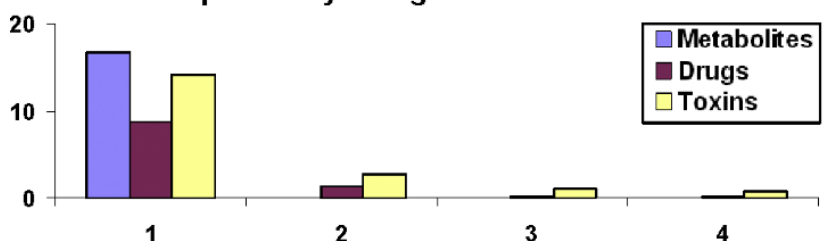

f. Number of negatively charged atoms

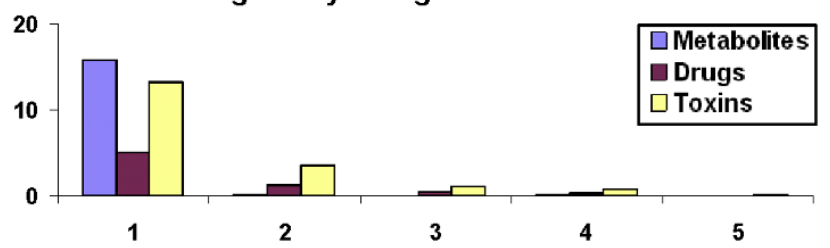

Figure 2

ID Atomic property differences between human metabolites, drugs and toxins. Compared properties include a. Number of atoms, b. Number of carbon atoms c. Number of nitrogen atoms d. Number of oxygen atoms e. Number of positively charged atoms f. Number of negatively charged atoms

the toxin dataset (91\%) contains $10-30$ atoms per molecule while only $9 \%$ of toxin molecules contain 30 or more atoms per molecule. The overall statistics of the three datasets is given in Table 2 and show that metabolites tend to have more atoms than drugs and toxin molecules.

\section{Carbon content}

Almost half of the molecules in the metabolite dataset have carbon atoms in the range 35-55 while 32\% have 5-25 carbon atoms per molecule (Fig. 2b). The carbon atom distribution in metabolites has a mean of 33 atoms and a maximum of 100 . On the other hand, drugs have a mean of 18 carbon atoms per molecule, with a maximum of 256 and $76 \%$ of drugs have carbon atoms in the range 5-25. Similar to drugs, toxin molecules also seem to prefer fewer carbons. In the toxin dataset, $77 \%$ of the molecules have 5-25 carbon atoms, while $16 \%$ have five or fewer carbon atoms. Only $7 \%$ of the molecules have more than 25 carbon atoms in toxin dataset. The distribution of carbon atoms in the toxin dataset has a mean of 12 and a maximum of 62 . From Table 2, we note that metabolites contain more carbon atoms than drugs, which in turn have greater carbon content than toxin molecules.

\section{Nitrogen content}

Approximately $40 \%$ of metabolites do not have any nitrogen atom (Fig. 2c), while 45\% have only one nitrogen atom and $16 \%$ have two or more nitrogen atoms per molecule. In sharp contrast to metabolites, only $15 \%$ of drug molecules do not posses nitrogen atoms while $74 \%$ of the molecules have nitrogen atoms in the range $1-5$. On the other hand, 30\% of the toxin molecules are devoid of any nitrogen atom while $66 \%$ of 
a. Number of rings

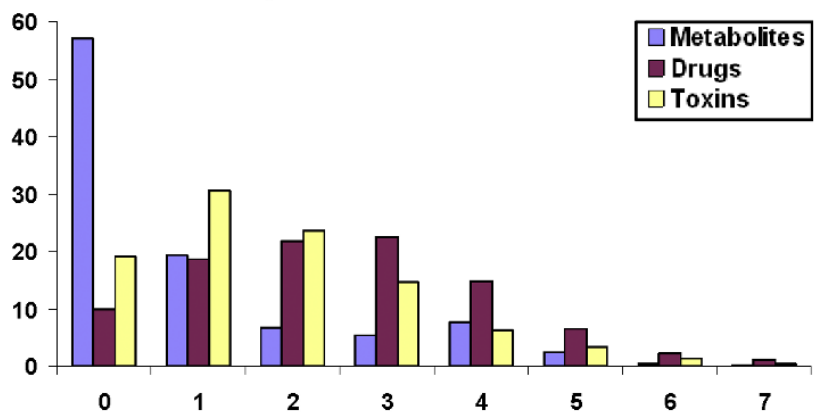

c. Number of rotatable bonds

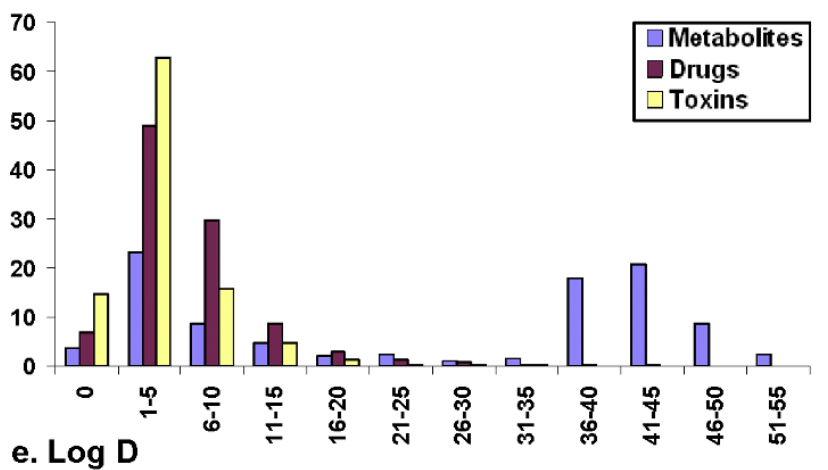

e. $\log D$

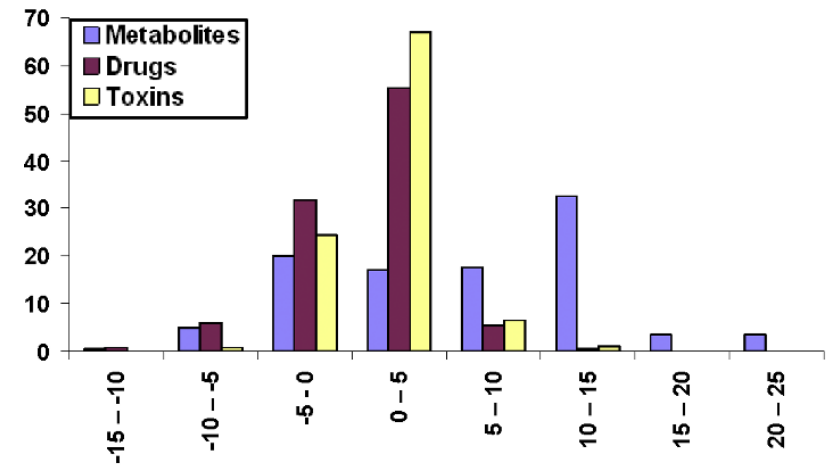

b. Number of ring assemblies

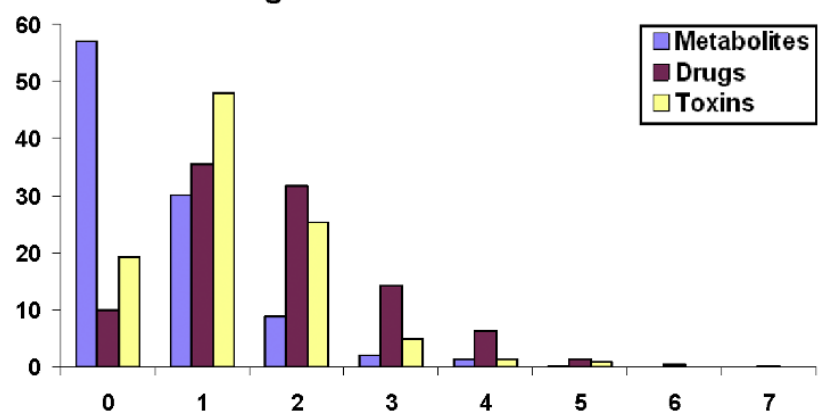

d. Number of aromatic bonds

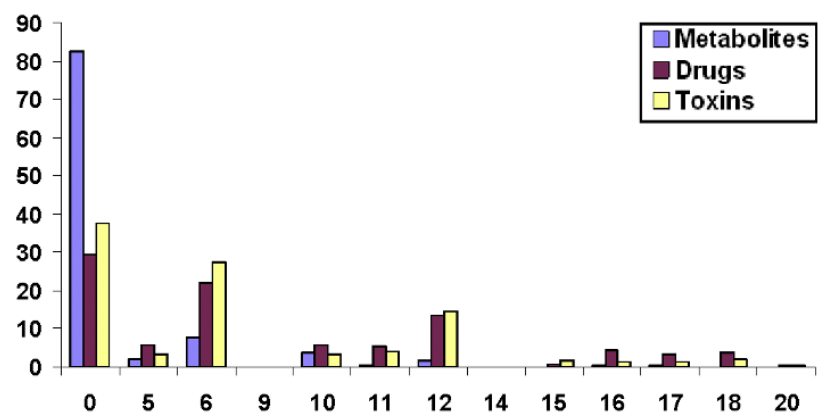

\section{f. Molecular solubility}

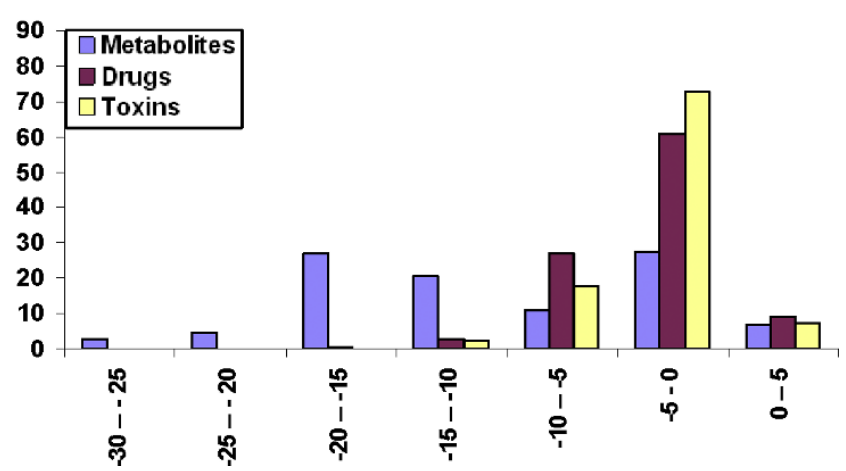

Figure 3

Other ID properties compared among human metabolites, drugs and toxins. Compared properties include a. Number of rings, b. Number of ring assemblies c. Number of rotatable bonds, d. Number of aromatic bonds, e. Log D, f. Molecular solubility.

toxin molecules contain nitrogen atoms in the range 1-5 and only $3 \%$ have six or more nitrogen atoms. From Table 2 and the values presented above, drugs molecules clearly possess the most number of nitrogen atoms, followed by toxin molecules and lastly, metabolites.

\section{Oxygen content}

For the three datasets, there is a clear reversal of the trend for the oxygen atom distribution compared to the nitrogen atom distribution presented in the previous section. Only $1 \%$ of the metabolite molecules do not have an oxygen atom as compared to $8 \%$ of drugs and $15 \%$ of toxin molecules (Fig. 2d). Furthermore, in metabolite dataset, $73 \%$ of the molecules possess oxygen atoms in the range $2-8$, compared to $68 \%$ of drugs and $65 \%$ of the toxins. Metabolites comprise more oxygen atoms than drugs, followed by toxic compounds, with mean values of eight, five and three, respectively (Table 2).

Number of negatively and positively charged atoms The fraction of molecules with a single negatively charged atom in the metabolite dataset $(16 \%)$ is almost 
a. Molecular surface area

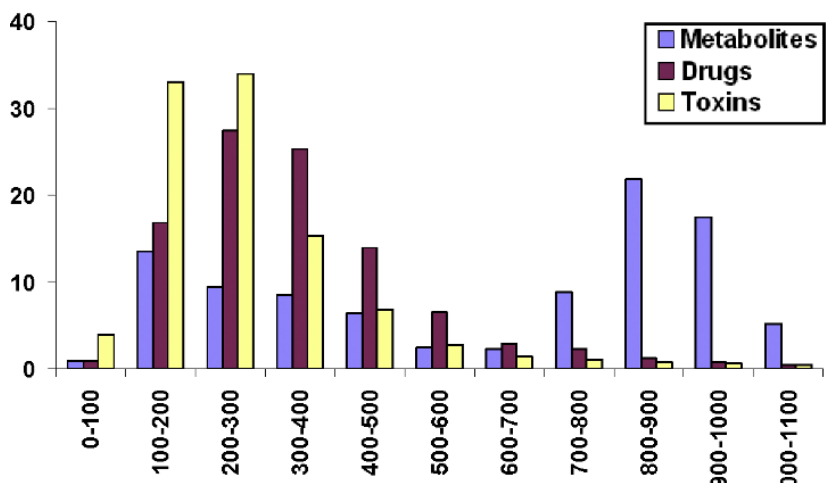

c. Molecular polar surface area

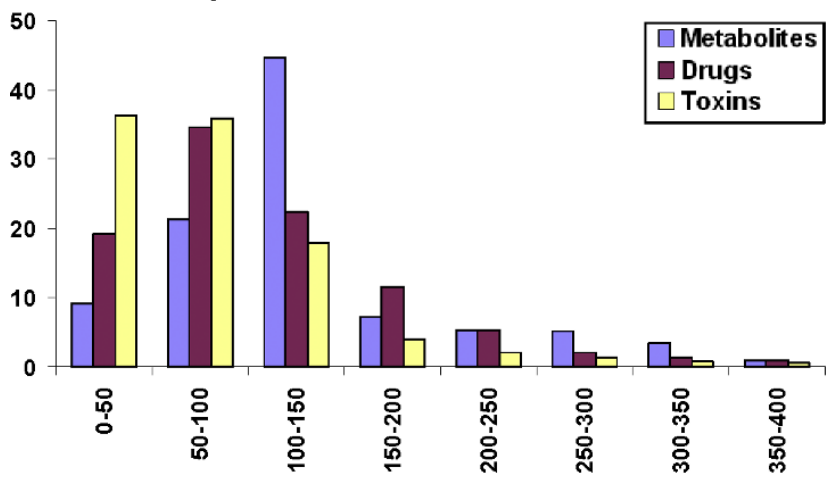

e. Molecular polar solvent accessible surface area

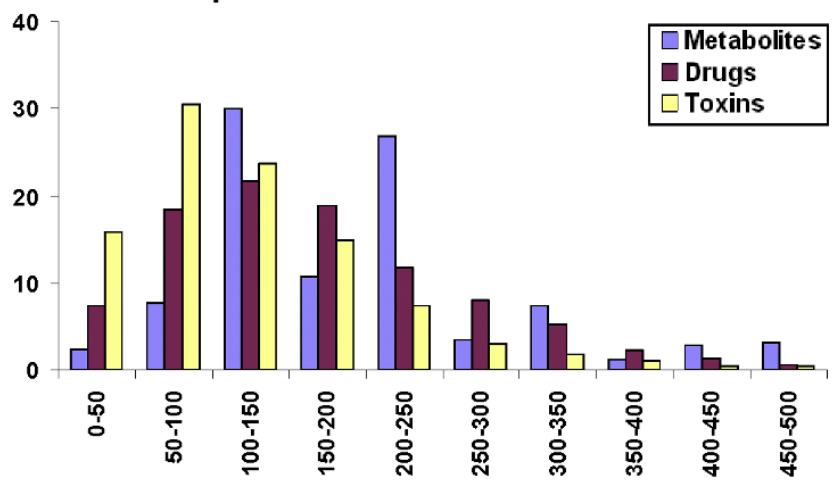

\section{b. Molecular volume}

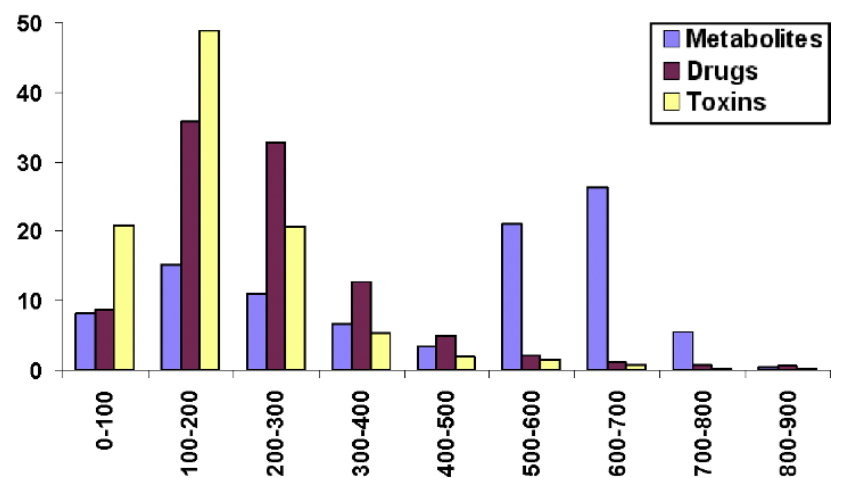

d. Molecular solvent accessible volume

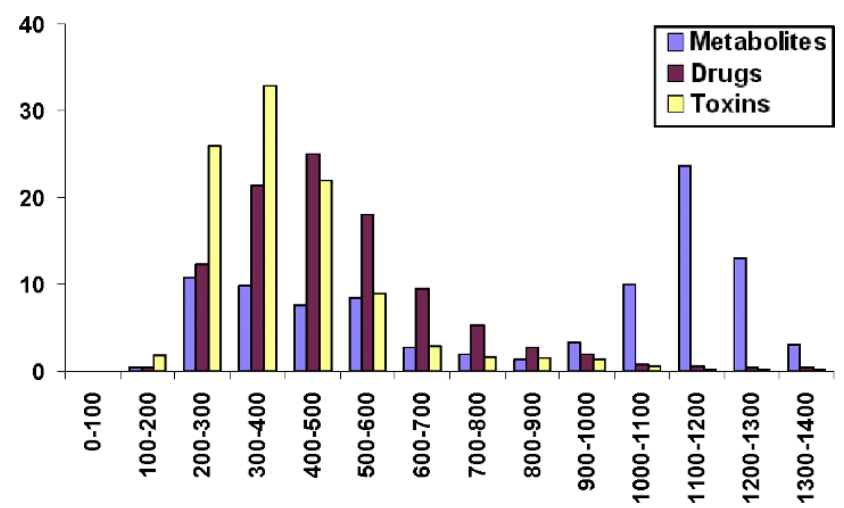

f. Molecular solvent accessible surface area

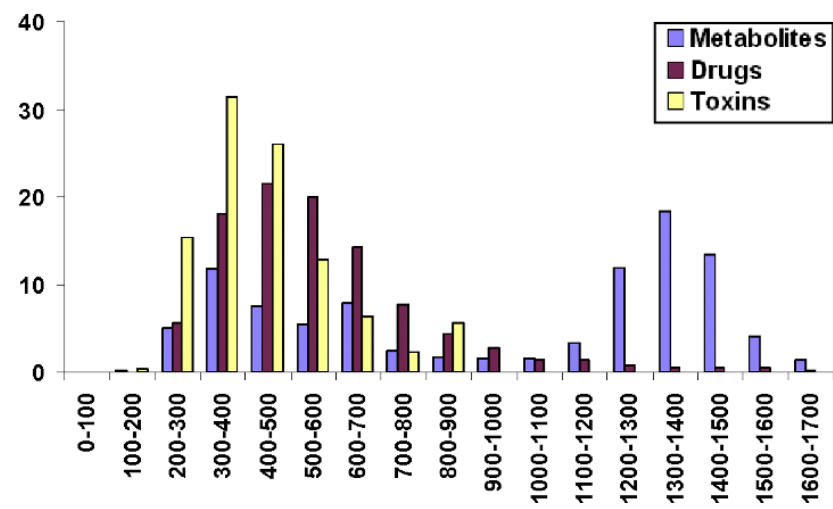

Figure 4

Comparison of 3D properties among human metabolites, drugs and toxins. Compared properties include a. Molecular surface area, b. Molecular volume c. Molecular polar surface area, d. Molecular solvent accessible volume, e. Molecular polar solvent accessible surface area, f. Molecular solvent accessible surface area.

the same as that containing one positively charged atom $(17 \%)$. While the metabolite dataset contains molecules with more than one negatively charged atom, there are no molecules with more than one positively charged atom. The percentage of negatively charged atoms is smaller in the drug dataset as compared to metabolite dataset (Fig. 2e and Fig. 2f). Only 5\% of drug molecules contain a negatively charged atom, with only $2 \%$ containing two or more negatively charged atoms, whereas $8 \%$ contain one positively charged atom. On the other hand, in the toxin dataset, $13 \%$ of the molecules contain one negatively charged atom per molecule and $5 \%$ of molecules contain two or more negatively charged atoms, whereas $14 \%$ of the molecules in the same dataset contain one positively charged atom per molecule and $4 \%$ contain two or higher positively charged atoms. The trend of charged atoms among the three datasets is Metabolites $>$ Toxin molecules $>$ Drugs, 


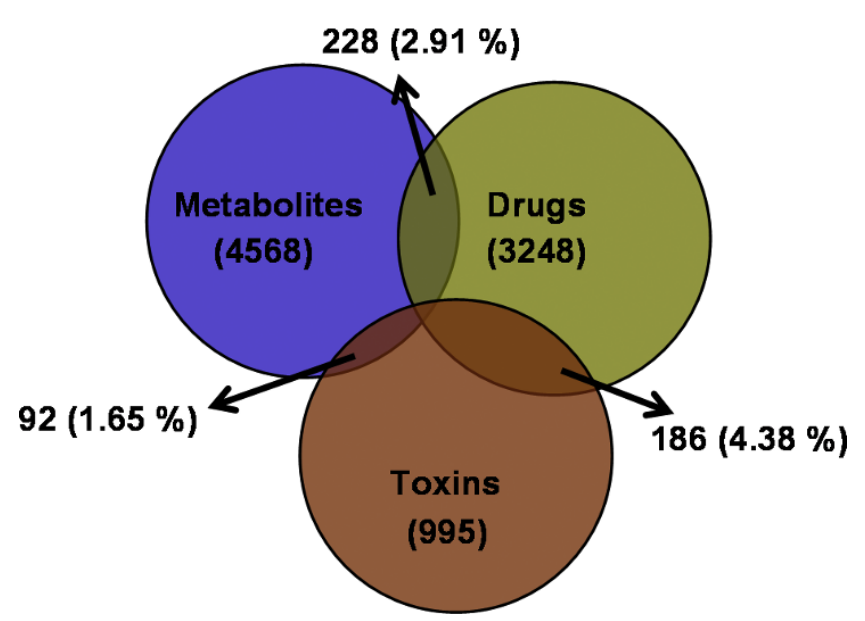

Figure 5

Venn diagram showing the overlap between the three clustered datasets.

with the drug dataset favouring negatively charged atoms over positively charged ones.

\section{Number of rings}

The distribution of number of rings is shown in Fig. 3a. Although more than $55 \%$ of the molecules in the metabolite dataset are acyclic, $19 \%$ contain a single ring and $21 \%$ contain $2-5$ rings. In sharp contrast to metabolites, only $9 \%$ of the drugs are acyclic, while almost $60 \%$ contains rings, with a three-way distribution ( $20 \%$ each) between one, two and three rings per molecule. The remaining $23 \%$ of drug molecules contains 4-6 rings, the maximum number of rings being 38. In the toxin dataset, $19 \%$ of the molecules are acyclic, whereas $68 \%$ contain 1-3 rings per molecule. The remaining $10 \%$ of toxins contain four or more rings per molecule. Thus, the pattern of ring distribution among the three datasets is Drugs > Toxin molecules > Metabolites.

\section{Number of ring assemblies}

After removing the non-ring bonds from a molecule the remaining backbone is termed as the ring assembly. As shown in the Fig. 3b, more than half of the molecules $(57 \%)$ in the metabolite dataset have no ring assembly, while $30 \%$ of the molecules have one ring assembly and $13 \%$ have two or more ring assemblies per molecule. On the other hand, in the drug dataset, only $10 \%$ of the molecules are free of ring assembly, whereas 36\% and $32 \%$ have one and two ring assemblies, respectively. Furthermore, in the same dataset, 23\% molecules contain more than three ring assemblies per molecule. Similar to drugs, most of the toxins possess ring assemblies with only $19 \%$ are devoid of any ring assembly, while $45 \%$ molecule have a single ring assembly. The percentage of molecules with two ring assemblies in the same dataset is $21 \%$ whereas $6 \%$ of the molecules have three or more ring assemblies. The pattern of ring assemblies is similar to that obtained for ring distribution in three datasets, being Drugs $>$ Toxin molecules > Metabolites.

\section{Number of rotatable bonds}

The number of rotatable bonds is a measure of molecular flexibility and is important in determining oral bioavailability of the drugs [30]. Only $4 \%$ of the molecules in the human metabolite dataset have no rotatable bonds, whereas 32\% have 1-10 rotatable bonds and $47 \%$ of the molecules have rotatable bonds in the range 36-50 (Fig. 3c). The mean value for rotatable bond distribution in metabolite dataset is 27 , with the maximum number of rotatable bonds in a metabolite molecule being 83 (Table 2). Among the drug molecules, $7 \%$ are devoid of rotatable bonds, while $79 \%$ of molecules have 1-10 rotatable bonds. Another 12\% of the molecules in this dataset have rotatable bonds in the range of 10-20. The mean value for rotatable bonds per molecule in drugs is 6 , with a maximum of 170 . In contrast to metabolites and drugs, $15 \%$ of toxin molecules do not possess any rotatable bonds, while $79 \%$ of the molecules contain rotatable bonds in the range of 1-10. The mean value for rotatable bonds in toxin molecules is 3 and the maximum number of rotatable bonds in a toxin dataset is 31. Thus, metabolites are more flexible than drugs and toxins.

\section{Number of aromatic bonds}

More than $80 \%$ of metabolites do not possess any aromatic bond. The remaining metabolites have several aromatic bonds, usually as multiples of five or six. As shown in Fig. 3d, 6\% of the molecules have either five or ten aromatic bonds, while $8 \%$ of molecules have either six or twelve aromatic bonds. The maximum number of aromatic bonds in metabolites is 36 .

In contrast to metabolites, only $29 \%$ molecules of drugs have no any aromatic bonds. Of the remaining, $12 \%$ and $36 \%$, respectively, of drug molecules have aromatic bonds as multiple of five and six. The maximum number of aromatic bonds in the drug dataset is 62 . On the other hand, toxin molecules are predominantly aromatic $(61 \%)$ with $7 \%$ and $42 \%$ having aromatic bonds as multiples of five and six, respectively. The maximum number of aromatic bonds in the toxin dataset is 46 . The order of aromatic bond distribution is Drugs $\approx$ Toxin molecules > Human metabolites, with almost half the aromatic bonds in all the three datasets being multiples of five or six. 
Log D

For solutes that can ionize, the distribution coefficient (D) is the ratio of the sum of the concentrations of all forms of the compound (ionized plus un-ionized) in each of the two solution phases. $\log \mathrm{D}$ is thus considered a better measure of lipophilicity that Alog P. However, for all three datasets, $\log \mathrm{D}$ follows the same distribution as Alog P (Fig 3e and Table 2).

\section{Molecular solubility}

Human metabolites have large range of molecular solubility values for example more than $85 \%$ of the molecules in metabolite dataset have molecular solubility in the range of -20 to 0 . Drug molecules have a smaller range of molecular solubility as compared to metabolites, with $87 \%$ of the molecules in drug dataset having molecular solubility values spanning -10 to 0 . Similarly, more than $90 \%$ of toxin molecules have a solubility value in the range -10 to 0 . Thus, the most preferred and common range of molecular solubility among the three datasets is -10 to 0 , which comprises $\sim 85 \%$ of the drug and toxin datasets but only $38 \%$ of the metabolite dataset (Fig. $3 \mathrm{f}$ and Table 2). The molecular solubility of metabolites is more than that of drugs, followed by toxin molecules, which suggests that metabolites tend to dissolve more easily than drugs and toxins in vivo (aqueous media).

\section{Chirality}

Chirality seems to be a distinguishing feature among the three datasets. The majority of the molecules in the metabolite dataset (74\%) are chiral. Chirality falls sharply in drugs and toxic compounds to $31 \%$ and $14 \%$, respectively.

Number of halogen atoms per molecule

As expected, toxin molecules have the highest number of halogen atoms per molecule compared to metabolites and drugs. $31 \%$ of molecules in toxin dataset possess a single halogen atom $(\mathrm{F}, \mathrm{Cl}, \mathrm{Br}, \mathrm{I})$ per molecule while in case of drugs close to $18 \%$ contain halogen atoms. In sharp contrast, to these two datasets, metabolites have far fewer halogen atoms per molecule. Only 15 out of 4568 molecules, i.e. only $0.3 \%$ of the molecules, studied are reported to have any halogen atom. The trend for halogens is Toxin molecules $>$ Drugs $>>$ Metabolites. The statistics provided in Table 3 provides information on the number of halogen containing molecules in each dataset.

Number of Sulphur and Phosphorus atoms per molecule Only $5 \%$ of the molecules in metabolite dataset, $20 \%$ of the drugs and almost the same percentage $(16 \%)$ of toxin molecules contain one or more sulphur atoms in their molecule. For sulphur atoms, the trend is Drugs $\approx$ Toxin molecules $>$ Metabolites. The trend gets reversed in the case of phosphorus atoms, with $46 \%$ of molecules in metabolites, $13 \%$ of drug molecules and only $3 \%$ of the toxic dataset having one or more phosphorus atoms. So the trend in phosphorus atom distribution is Metabolites $>>$ Drugs > Toxin molecules.

\section{Average bond length per molecule}

The metabolite molecules form two groups, with $48 \%$ having a mean value of $0.82 \AA$ bond length, while another $51 \%$ have $0.83 \AA$. The majority of drug molecules $(65 \%)$ also have either 0.82 or $0.83 \AA$ bond length. In sharp contrast to the other two datasets, the average bond length for $92 \%$ of molecules in toxic dataset is $1.33 \AA$, while another $7 \%$ of the molecules have $1.40 \AA$ as the average bond length. As far as average bond length is concerned, metabolites and drugs have a much shorter average bond length compared to toxin molecules, the trend being Toxin molecules > Metabolites $\approx$ Drugs.

\section{D Descriptors: molecular volume and surface area}

Molecular surface area

Molecular surface area distribution in metabolites is bimodal (Fig. 4a) with the first smaller peak at 100-400 $\AA^{2}$, containing $37 \%$ of the molecules and the second larger peak at 700-1100 $\AA^{2}$, with $53 \%$ of the molecules. On the other hand, $83 \%$ of drugs molecules have molecular surface area between 100-500 $\AA^{2}$. A similar distribution is obtained for toxin molecules with $89 \%$ of the compound in the toxin dataset having a molecular surface area in the range 100-500 $\AA^{2}$ and only $4 \%$ are in the range 0-100 $\AA^{2}$. From these values and the statistics in Table 2, metabolites have greater molecular surface area than drugs and toxin molecules.

Table 3: Halogen atom frequency distribution. The number of times different halogens are reported in each of the dataset is listed below

\begin{tabular}{lllll}
\hline Database & Fluorine & Chlorine & Bromine & lodine \\
\hline Metabolites & 15 & 32 & 0 & 27 \\
Drugs & 496 & 477 & 110 & 63 \\
Toxins & 62 & 473 & 38 & 5
\end{tabular}




\section{Molecular volume}

The results of molecular volume distribution in three datasets are reflected in the related property of molecular weight distribution. As depicted in Fig. 4b the molecular volume range in metabolites is much wider and in accordance with molecular weight data when compared to the other two datasets. Almost $47 \%$ of the molecules have molecular volume in the range of 500-700 $\AA^{3}$. The majority of molecular volume distribution of the drug dataset is narrow compared to that of human metabolites with $81 \%$ of the molecules are in the range form $100-400 \AA^{3}$, although the tails extend further, with some molecules found to have volumes above $1700 \AA^{3}$. The molecular volume range is even more restricted in toxic compounds with $90 \%$ of the molecules in the range 0-300 $\AA^{3}$ with $~ 49 \%$ of these having a molecular volume of $100-200 \AA^{3}$. So, the trend for molecular volume distribution is the same as that observed for molecular weight distribution among the three datasets: Metabolites $>$ Drugs $>$ Toxin molecules.

\section{Molecular polar surface area}

The polar surface area is defined as the surface area summed over all polar atoms, (usually oxygen and nitrogen), including the attached hydrogen atoms. It is often correlated with drug transport capabilities and is important for penetrating the blood-brain barrier (BBB). As most of the metabolites do not need to be shuttled through barriers like $\mathrm{BBB}$, they can afford to have more polar surface area than drugs and toxins. More than 95\% of the metabolites have polar surface area in the range $0-350 \AA^{2}$ (Fig. 4c) while $92 \%$ of polar surface area of drugs is contained within $0-250 \AA^{2}$. The distribution is even narrower for the toxin dataset with $90 \%$ of the molecules in the range 0-150 $\AA^{2}$.

Molecular solvent accessible volume

Molecular solvent accessible volume distribution is similar to the distribution of the molecular volume. In the case of metabolites (Fig. 4d), it also follows a bimodal distribution with a smaller peak of $36 \%$ molecules around 200-600 $\AA^{3}$ and a larger peak containing $46 \%$ of the molecules around 1000-1300 $\AA^{3}$. However, there is no molecule with accessible volume less than or equal to $100 \AA^{3}$. Unlike metabolites, drugs molecules have only one peak covering almost the entire dataset. About $91 \%$ of the drug molecules have solvent accessible volume from 200 to $800 \AA^{3}$. Like metabolites there is no molecule with solvent accessible volume less than or equal to 100 in drug dataset. The distribution of solvent accessible volume in toxin molecules is even thinner with $89 \%$ of the molecules in the range $200-600$ $\AA^{3}$. Other $7 \%$ are present in the range 600-1000 $\AA^{3}$. According to the statistics shown in Table 2 and Fig. 4 d, the order of molecular solvent accessible volume is Metabolites $>$ Drugs $>$ Toxin molecules.

Molecular polar solvent accessible surface area

Drugs and toxin molecules follow a perfect Gaussian distribution for polar solvent accessible surface area while metabolites follow a bimodal pattern (Fig. 4e). The maximum number of molecules in toxic dataset has molecular polar solvent accessible surface area is in the range 0-200 $\AA^{2}$ while for drugs the range is $0-350 \AA^{2}$. On the other hand maximum numbers of metabolites are covered in between 100-250 $\AA^{2}$. The statistics in Table 2 suggests that metabolites tend to have larger molecular polar solvent accessible surface area compared to drugs which in turn are larger than toxins.

\section{Molecular solvent accessible surface area}

Differences among metabolites, drugs and toxin molecules are readily observable for molecular solvent accessible surface area. Metabolites follow a bimodal distribution whereas drugs and toxins follow a Gaussian distribution (Fig. 4f). Toxin molecules peak at 300-500 $\AA^{2}$ while drugs peak at 400-600 $\AA^{2}$. Metabolites, on the other hand, form a lower peak at 300-400 $\AA^{2}$ with a second larger peak at 1200-1500 $\AA^{2}$. Form Table 2, metabolites have clearly larger values for molecular solvent accessible surface area than drugs and toxins.

\section{Functional group analysis}

The frequency of functional group occurrence among the three datasets was carried out in this study with the Scitegic Pipeline pilot software (details in the Methods section). The occurrence of specific functional groups of interest to drug design is given in Table 4 and Additional file 1 . Aromatic atoms are a prominent feature among drugs and toxins while only a sixth of metabolites have aromatic atoms. The same trend is observed in benzene ring distribution among the datasets. Further, primary and quaternary amines occur more frequently in metabolites than secondary and tertiary amines when compared to drugs and toxin molecules, respectively. Additionally, drugs are found to possess a greater number of amides than metabolites or toxins. Finally, toxic functional groups (like nitro, azo and cyanide) are only found in toxins while they are either absent or very limited in drugs and metabolites.

\section{Clustered vs. unclustered datasets}

We have compared all the above property distributions for clustered and unclustered (raw) datasets (data not shown). Correlation coefficients were calculated for all the properties and eight properties which are not significantly correlated are presented here, viz. Alog P, molecular weight, the number of oxygen atoms, the 
Table 4: Occurrence of functional groups in the three datasets

\begin{tabular}{lccc}
\hline Functional Group & $\begin{array}{c}\text { Metabolite } \\
\text { dataset }\end{array}$ & $\begin{array}{c}\text { Drugs } \\
\text { dataset }\end{array}$ & Toxin dataset \\
\hline Alkyl halide & $<0.5 \%$ & $<0.5 \%$ & $3.2 \%$ \\
Aromatic atom & $17.4 \%$ & $70.6 \%$ & $62.3 \%$ \\
Benzene & $10.3 \%$ & $56.0 \%$ & $53 \%$ \\
Steroid backbone & $2.9 \%$ & $0.6 \%$ & $<0.5 \%$ \\
HBA Ester & $56.3 \%$ & $13.8 \%$ & $15.4 \%$ \\
Pyridine & $1.2 \%$ & $6.4 \%$ & $5.3 \%$ \\
Pyrimidine & $3.2 \%$ & $7.5 \%$ & $1.9 \%$ \\
Enamine & $3.2 \%$ & $10.31 \%$ & $3.41 \%$ \\
Primary amine & $28 \%$ & $14.4 \%$ & $12.0 \%$ \\
Secondary amine & $11.4 \%$ & $64.0 \%$ & $41.2 \%$ \\
Tertiary amine & $44.6 \%$ & $80.0 \%$ & $60.0 \%$ \\
Quaternary Amine & $15.3 \%$ & $2.1 \%$ & $0.5 \%$ \\
Primary amide & $1.5 \%$ & $4.5 \%$ & $3.9 \%$ \\
Secondary amide & $11.4 \%$ & $31.0 \%$ & $14.5 \%$ \\
Tertiary amide & $2.8 \%$ & $16.8 \%$ & $9.2 \%$ \\
Imines & $4.1 \%$ & $14.0 \%$ & $6.4 \%$ \\
Azo & $0 \%$ & $<0.5 \%$ & $3.4 \%$ \\
Carbamic acid & $<0.5 \%$ & $3.1 \%$ & $1.9 \%$ \\
Urea & $2.5 \%$ & $8.0 \%$ & $6.5 \%$ \\
\hline
\end{tabular}

Those functional groups which can discriminate between the three datasets are presented here. The complete list is in Additional File I.

number of nitrogen atoms, molecular polar surface area, molecular solubility, the number of rings and the number of aromatic bonds (Figs. 6, 7, 8, 9). Alog P and molecular weight values (Fig. 6) do not deviate significantly with clustering. Nitrogen atom distribution (Fig. 7) for clustered and unclustered molecules also remains fairly similar for all the datasets. The analysis also shows that the number of aromatic bonds (Fig. 8) and the molecular solubility distribution (Fig. 9) are also fairly conserved between clustered and unclustered datasets. We note that, by and large, the two distributions are very similar except in following cases:

\section{Number of oxygen atoms}

There is an exception at five oxygen atoms per molecule in the unclustered metabolite dataset (Fig. 7b).

\section{Number of rings}

The number of molecules with zero rings drops for drugs ( $8 \%$ decrease) and toxins ( $9 \%$ decrease) whereas metabolites follow a similar distribution in clustered and unclustered dataset comparison (Fig. 8a).

\section{Molecular polar surface area}

Clustered metabolites show a $10 \%$ decrease in molecules with polar surface area in the range 50-100 $\AA^{2}$ while clustered toxins show a $15 \%$ increase in the number of molecules with polar surface area between 0 to $50 \AA^{2}$. Drugs, on the other hand, follow a similar distribution for clustered and unclustered datasets (Fig. 9a).

\section{Conclusion}

We have carried out a comprehensive analysis of three publicly available datasets, comprising drug, metabolite and toxin molecules. We have also, for the first time, to the best of our knowledge, compared the distributions of various properties for complete datasets (unclustered data) as well as reduced or clustered datasets. We note that, in the main, the distributions for the two data groups, clustered and unclustered, are very similar, supporting the use of clustered datasets, except in the case of the number of oxygen atoms, the molecular polar surface area and the number of rings. Based on this result, these properties should be treated with caution for lead discovery in drug discovery pipelines with unclustered datasets.

From the analyses of clustered datasets, we find that twothirds of the human metabolites lie outside the Lipinski universe. On the other hand, over $90 \%$ of the toxin molecules abide by Lipinski's rule, implying that since Ro5 does not explicitly take toxicity into account, present-day drugs are consequently similar to toxins than to metabolites.

Results from the analysis of $1 \mathrm{D}$ and 3D molecular properties consolidate our finding of drugs and toxins sharing a larger property space, than drugs and metabolites. 1D properties such as the total number of atoms advocate that metabolites are bulky, with more carbon and hydrogen atoms than drug and toxins. This is consistent with the idea that metabolites are produced at the required subcellular location and thus do not need to be transferred from one location to another. In order to design metabolite-like drugs, it would be beneficial to attempt alternative ways for drug delivery, since traditionally, drugs are required to pass through the bloodbrain-barrier, which limits the size of drug molecules. Considering the numbers of nitrogen and oxygen atoms, metabolites prefer oxygen over nitrogen containing groups. Above $50 \%$ of the metabolites are acyclic while only $9 \%$ of the drugs and $19 \%$ of the toxin molecules are acyclic. The number of rotatable bonds measuring molecular flexibility and consequently, oral bioavailability, suggests that metabolites are far more flexible than drugs and toxin molecules. Over $70 \%$ of the drugs and $62 \%$ of toxin molecules are aromatic while only $20 \%$ of the metabolites are aromatic. This result is in accordance with the fact that drugs are derived from various sources including NPs which are mostly aromatic in nature. In all the datasets examined, the majority of molecules have negative solubility values, suggesting that a large proportion of these compounds are soluble in aqueous solutions. Chirality falls sharply from metabolites to drugs and toxin molecules while as expected, the number of halogen atoms are found to 

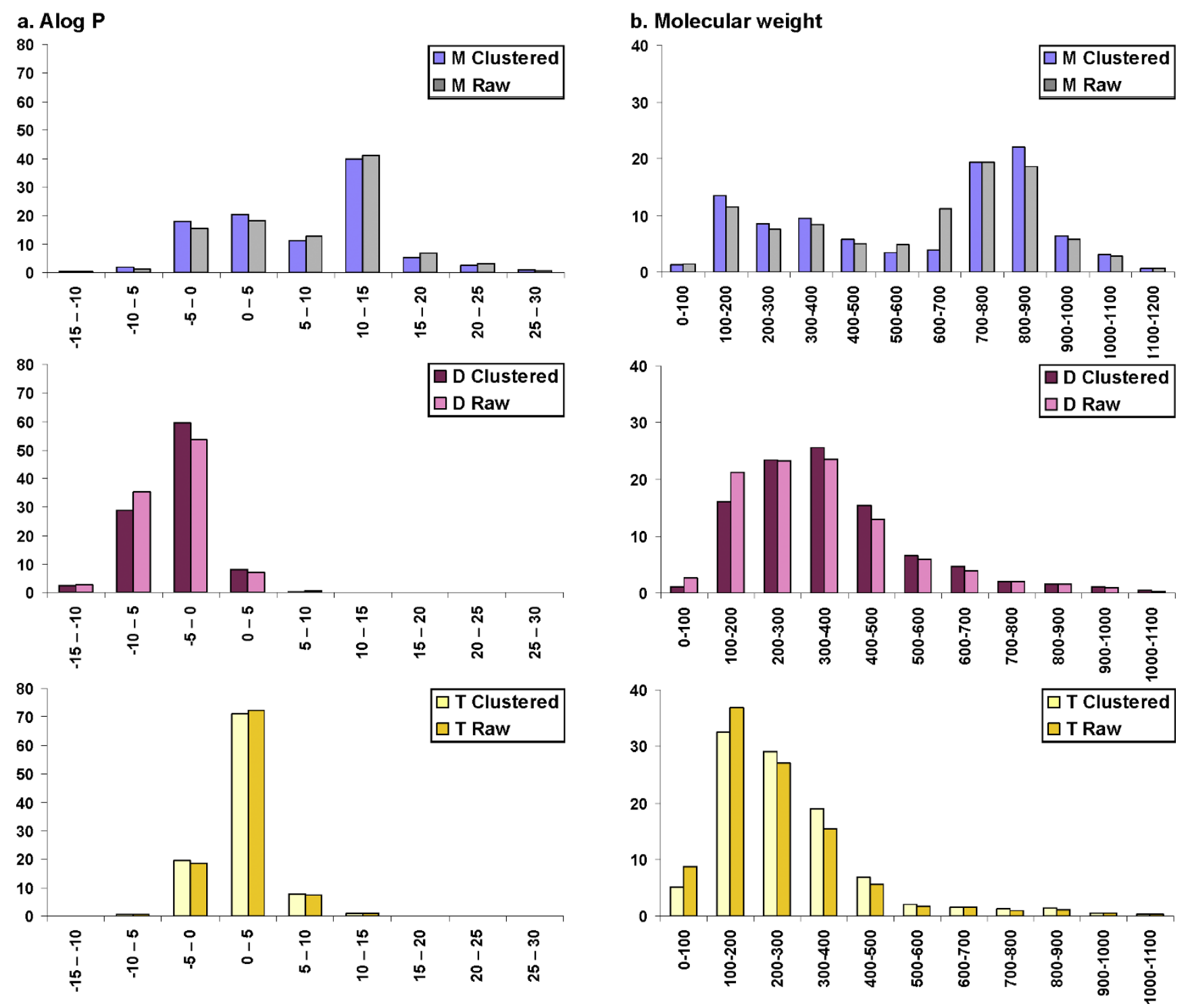

Figure 6

Comparison of example Lipinski properties for clustered and unclustered (raw) data. Properties compared are a. Alog P, b. Molecular weight, for human metabolites (M), drugs (D) and toxin molecules (T).

be higher in toxins than in drugs and metabolites. The average bond length of $90 \%$ metabolites and more than $65 \%$ of drugs is much smaller than majority of toxins, suggesting multiple bonds in the former datasets. The analysis results from 3D descriptors such as molecular volume and molecular surface area are reflected in the related property of molecular weight and confirm that present day drugs are more like toxins than metabolites.

The analysis also shows that although drugs share a relatively larger property space with toxins than with metabolites, drugs and toxins are two different classes of compounds as reflected in specific physicochemical characteristics. Drugs tend to have higher values for properties such as molecular weight, the number of oxygen atoms, the number of rotatable bonds and molecular polar surface area whereas toxin molecules have considerably higher Alog P and Log D values.

Additionally, empirical rules like the "rule of five" can be refined to increase the coverage of drugs or drug-like molecules that are clearly not close to toxic compounds, because toxicity reduction is one of the key aspects of drug discovery programs. Our results have implications 
a. Number of nitrogen atoms
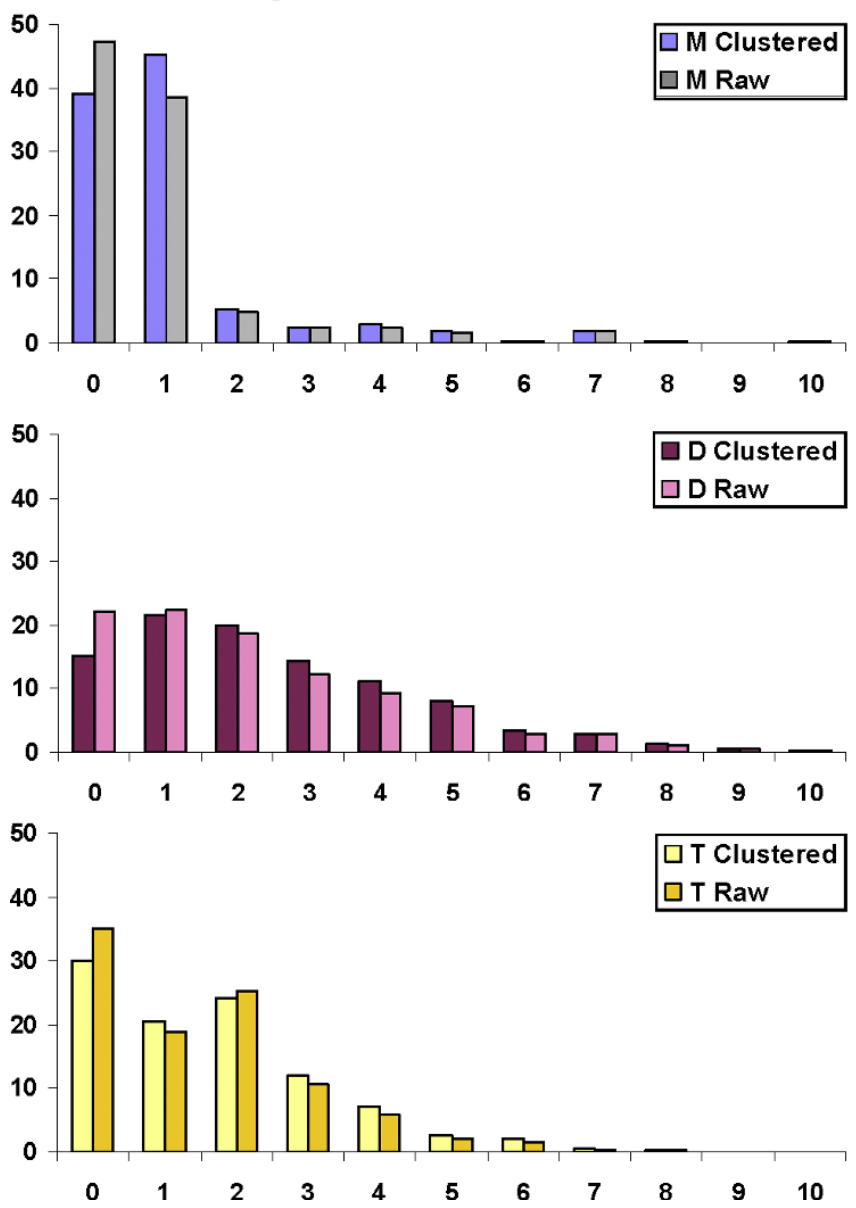

b. Number of oxygen atoms
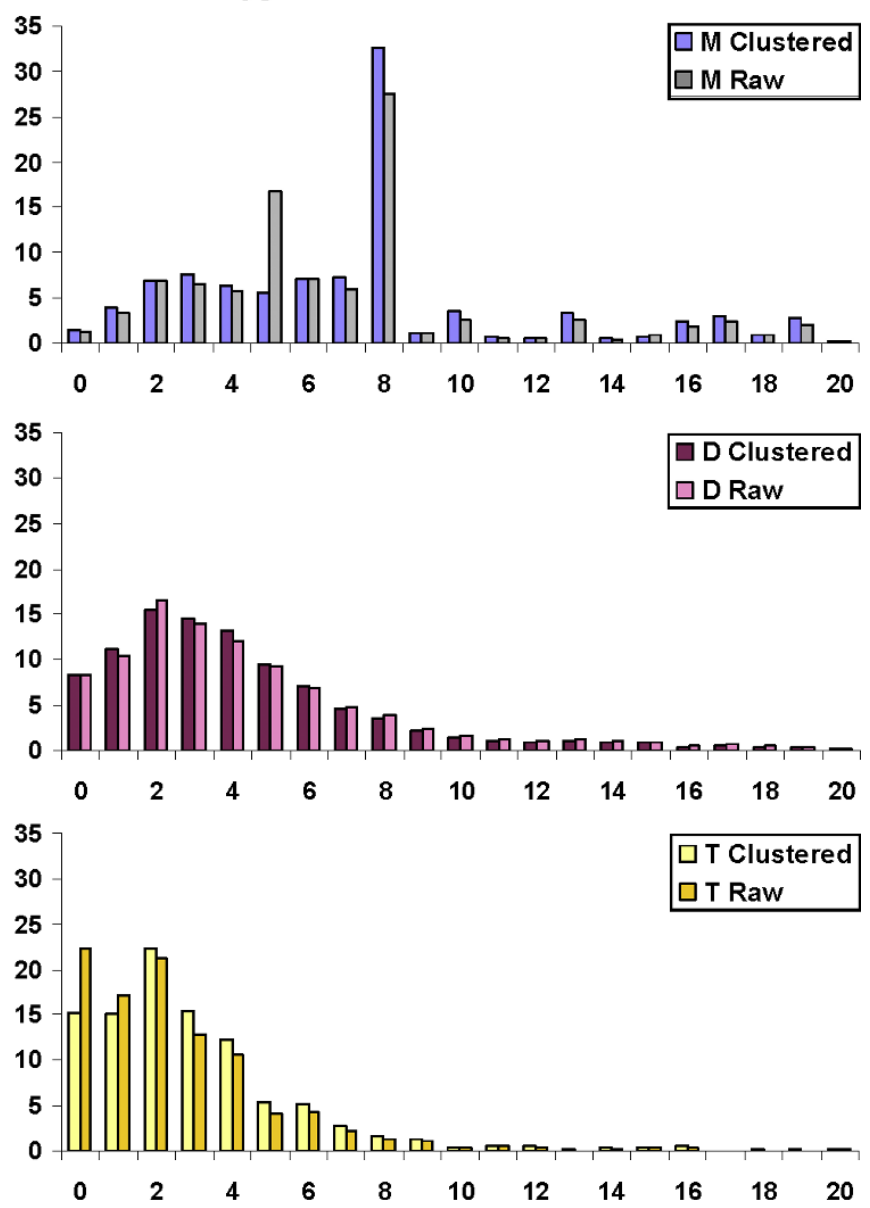

Figure 7

Comparison of example ID atomic properties for clustered and unclustered (raw) data. Properties compared a. Number of nitrogen atoms, b. Number of oxygen atoms, for human metabolites (M), drugs (D) and toxin molecules (T).

for the analysis of novel compounds in lead discovery pipelines, to uncover novel target molecules.

\section{Methods}

\section{Preparation of the dataset}

Three publicly available databases, relevant to human diseases and their treatment have been used in this study. The human metabolome database [31] contains information on nearly 7000 small molecule metabolites found in human body. Similarly, DrugBank [32] is a comprehensive resource on drugs and drug targets, with detailed chemical, pharmaceutical and medical information on nearly 3000 drug targets and 4800 drugs including $>1,350$ FDA-approved small drugs and experimental drugs derived from the PDB-Ligand database [33], containing compounds bound to biomolecules. Distributed Structure-Searchable Toxicity (DSSTox)
Carcinogenic Potency Database [34] is hosted by the US Environmental Protection Agency's National Center for Computational Toxicology aiming to provide a public data repository on toxicity data. DSSTox contains experimental results and carcinogenicity information for 1547 substances tested against different species.

Preliminary datasets containing 6668 human metabolites from the human metabolome database (as on 23-Dec2008), 4883 drugs from DrugBank (as on 6-Jan-2009) and 1547 toxin molecules from DSSTox (as on 16-Jan-2009) were extracted.

From these preliminary datasets duplicates and inorganic molecules (individual atoms, metal salts, inorganic oxides, hydroxides, cations and anions) were removed. Any "missing" compounds (either with no or incomplete structure) were also removed. The "cleaned" collections of 
a. Number of rings
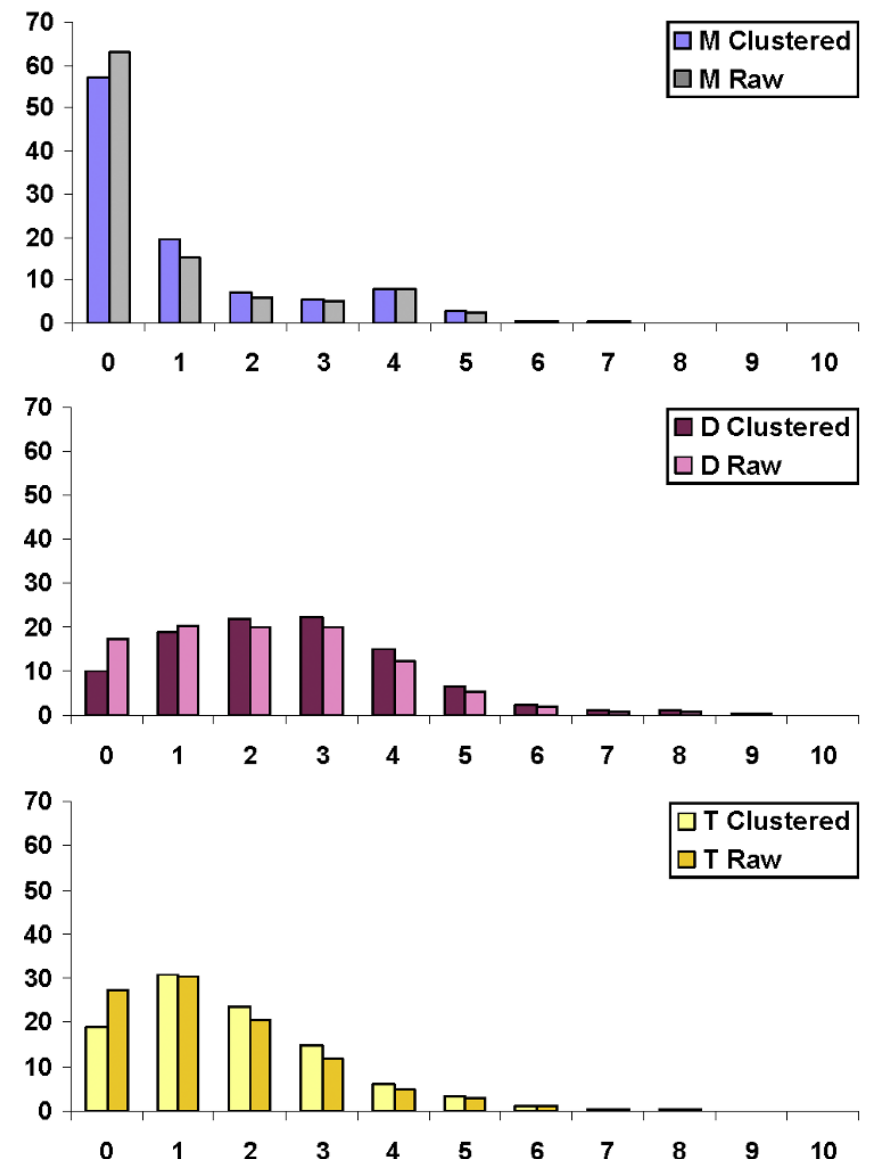

b. Number of aromatic bonds
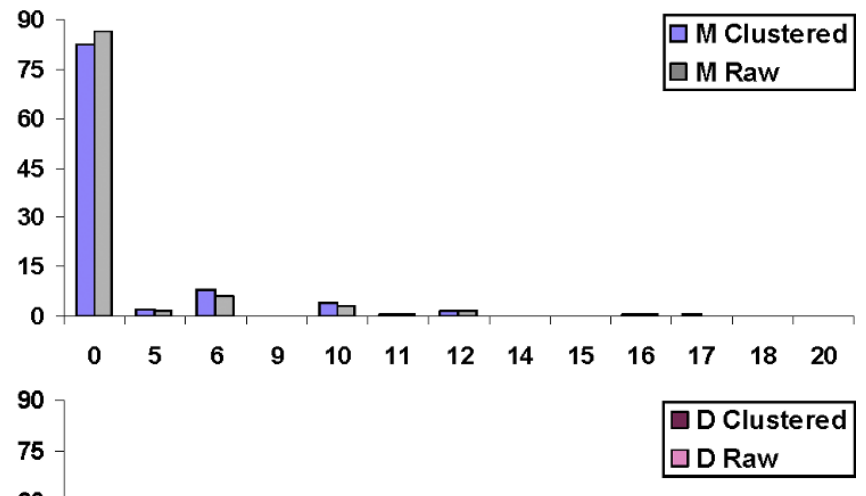

60
45
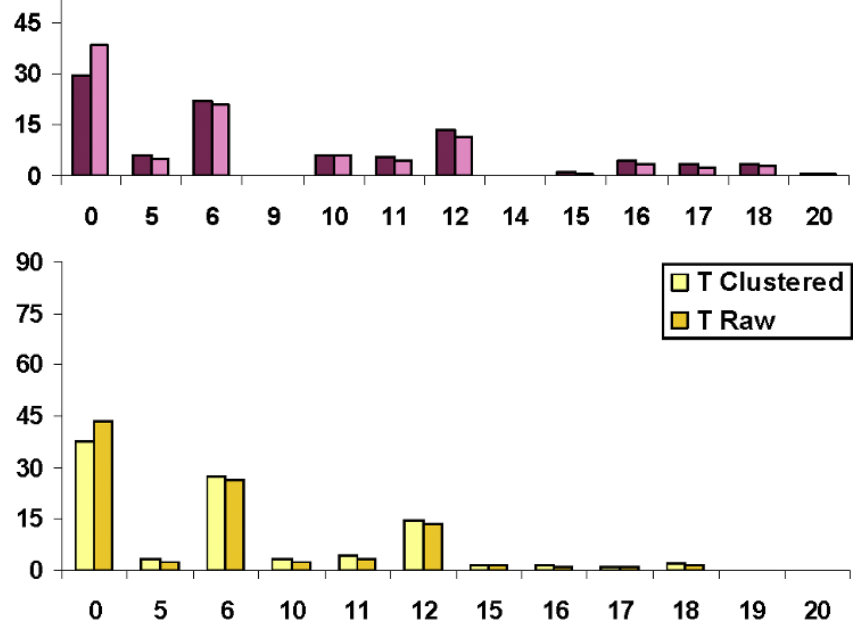

Figure 8

Comparison of example ID aromatic properties for clustered and unclustered (raw) data. Properties compared are a. Number of rings, b. Number of aromatic bonds, for human metabolites (M), drugs (D) and toxin molecules (T).

unique compounds were compiled into analysis datasets containing 6582 metabolites, 4829 drug molecules and 1448 toxin molecules. Finally, clusters were generated from each dataset, using the Cluster "Clara" algorithm embedded in the Scitegic Pipeline Pilot software [35], which is an approximate version of "partitioning around medoids" (pam) method comprising $70 \%$ of the entire raw data, similar to that reported in Dobson et al. [25]. Clustering was performed to address the issue of possible overrepresentation of the chemical space, which might bias the analysis results towards these redundant molecules. Representative sets of molecules were produced by employing the extended connectivity fingerprint (ECFP) [36,37] as a molecular descriptor and Euclidean distance was the distance metric selected. ECFP generates an array of structural features by encoding each atom and its molecular environment within a sphere of specified diameter. Cluster centres were selected as the representatives, for clusters containing more than one molecule while singletons were directly used as cluster centres in non-cluster situations. The contents of unclustered and clustered datasets, prepared for analyses are presented in Table 5.

The overlap among the three clustered datasets (CM, CD and CT) was calculated and it was found that more compounds are common in between drugs and toxin molecules than any other combination. The results are displayed in Figure 5. As the binary overlap is very small $(<5 \%)$ and the ternary overlap is negligible, the datasets were retained as such, without further size reduction.

\section{Calculations of the physicochemical properties}

The calculation of all the molecular properties was carried out through the Scitegic pipeline pilot [35] and in-house Perl scripts.

Two types of hydrogen bond acceptors and donors were taken into account. Firstly, the Lipinski type donors (sum 
a. Molecular polar surface area
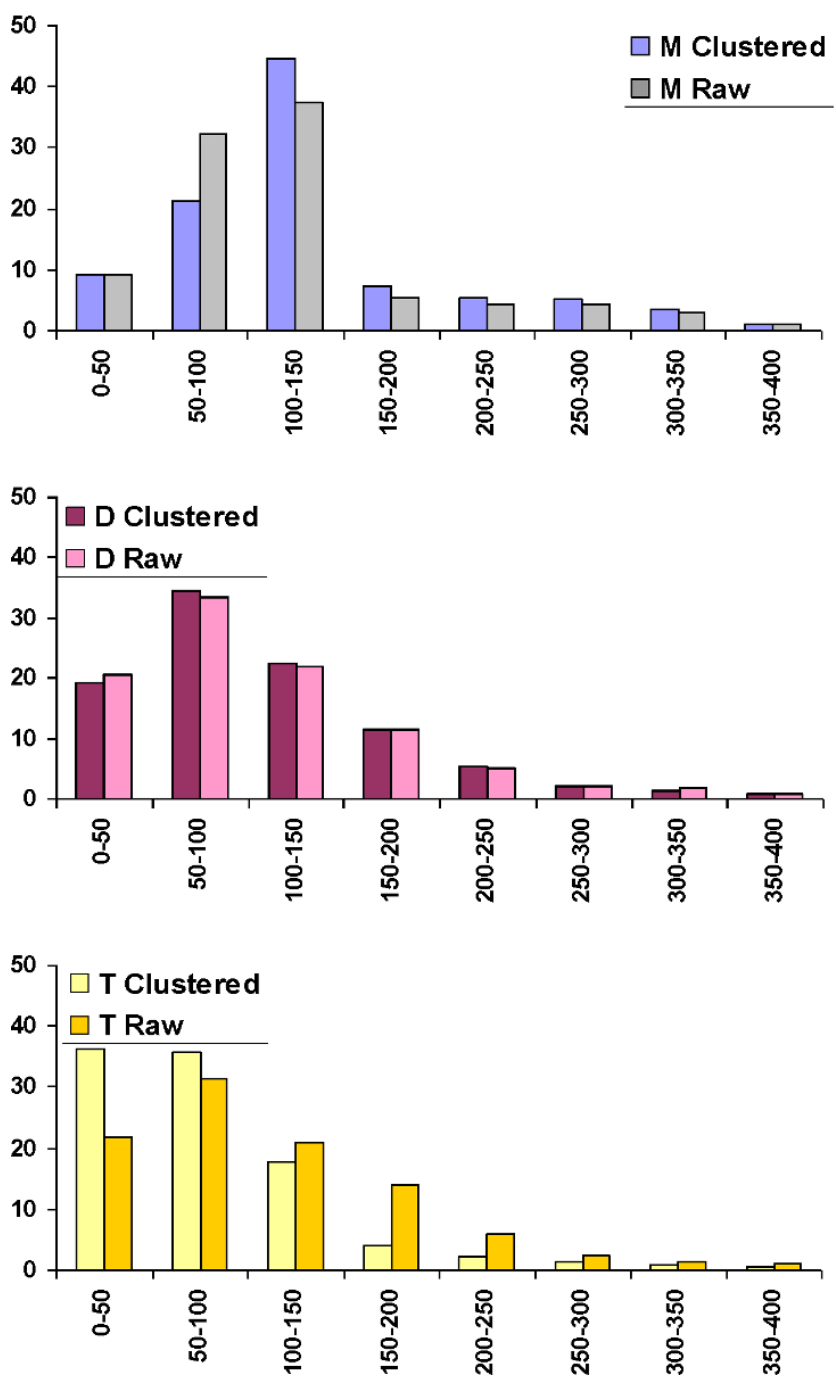

\section{b. Molecular solubility}
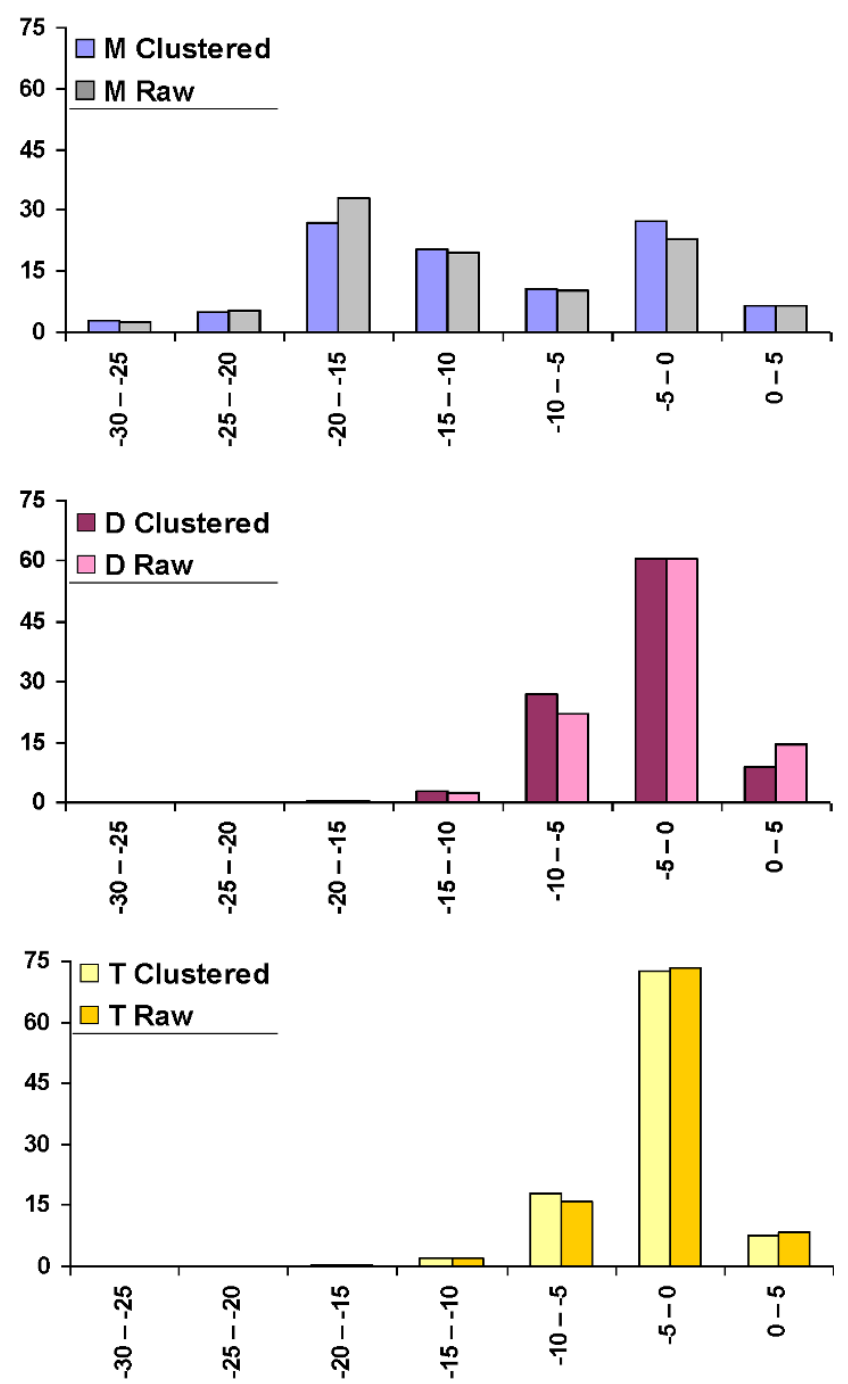

Figure 9

Comparison of example molecular properties important in drug design, for clustered and unclustered (raw) data. Properties compared are a. Molecular polar surface area, b. Molecular solubility, for human metabolites (M), drugs (D) and toxin molecules $(\mathrm{T})$.

Table 5: Clustered and Unclustered datasets

\begin{tabular}{llll}
\hline Dataset & Metabolites & Drugs & Toxin molecules \\
\hline Unclustered & M: 6582 & D: 4829 & T: 1448 \\
Clustered & CM: 4568 & CD: 3248 & CT: 995 \\
\hline
\end{tabular}

of $\mathrm{OH}$ and $\mathrm{NH}$ ) and acceptors (sum of $\mathrm{N}$ and $\mathrm{O}$ atoms) were calculated as defined by Lipinski et al. [3] and then, all available hydrogen bond donors and acceptors were summed up.
The octanol-water partition coefficient was either retained if provided with the data, or was calculated from Scitegic software. The hydrophobicity measure, Alog P, was calculated using the Ghose-Crippen method [38] which takes into account the group contribution to Log P. Another partition coefficient, Log D (the distribution coefficient), which take into account unionized and ionized species, was also calculated. Log D is equal to $\log \mathrm{P}$ for unionizable compounds but with ionized species, $\log \mathrm{D}$ is considered better than $\log \mathrm{P}$, as it takes ionized species into account, along with unionized forms. A positive value of Log P or Log D suggests a 
preference to lipophilic surroundings, whereas a negative value indicates preference to lipophobic (or hydrophilic) environment.

$$
\log \mathrm{D}=\sum\left[\mathrm{C}_{\mathrm{i}}\right]_{\text {oct }} / \sum\left[\mathrm{C}_{\mathrm{i}}\right]_{\mathrm{aq}}
$$

Other simple count-based molecular descriptors enumerating aromatic bonds, atoms, carbon atoms, nitrogen atoms, oxygen atoms, hydrogen atoms and rings were also calculated. Beside these, one-dimensional (1D) descriptors calculated include molecular weight and molecular solubility. Three-dimensional (3D) descriptors like molecular volume, molecular surface area, molecular polar surface area and molecular solvent accessible surface area were also computed. The molecular polar surface area is defined as the sum of all the polar atoms (usually oxygen and nitrogen atoms, and the attached hydrogen atoms). This descriptor is often correlated with drug transport capabilities and is important in penetrating the blood-brain barrier.

\section{Competing interests}

The authors declare that they have no competing interests.

\section{Authors' contributions}

VK curated the dataset and conducted the analysis work; SR directed the study and both authors prepared and approved the manuscript.

\section{Note}

Other papers from the meeting have been published as part of BMC Genomics Volume 10 Supplement 3, 2009: Eighth International Conference on Bioinformatics (InCoB2009): Computational Biology, available online at http://www.biomedcentral.com/1471-2164/10? issue $=\mathrm{S} 3$.

\section{Additional material}

\section{Additional file 1}

Table S1. Occurrence of discriminatory functional groups in the three datasets.

Click here for file

[http://www.biomedcentral.com/content/supplementary/14712105-10-S15-S10-S1.pdf]

\section{Acknowledgements}

We thank Assoc. Prof. J. Jamie for useful discussions during this study. VK is grateful to Macquarie University for the award of MQRES research scholarship. Open access publication charges for this article were covered by Macquarie University.
This article has been published as part of BMC Bioinformatics Volume 10 Supplement 15, 2009: Eighth International Conference on Bioinformatics (InCoB2009): Bioinformatics. The full contents of the supplement are available online at http://www.biomedcentral.com//47I-2/05//0? issue $=\mathrm{S} 15$.

\section{References}

I. Hodgson J: ADMET - turning chemicals into drugs. Nat Biotechnol 200I, 19(8):722-726.

2. Lipinski $C$ and Hopkins A: Navigating chemical space for biology and medicine. Nature 2004, 432(7019):855-86I.

3. Lipinski CA, Lombardo F, Dominy BW and Feeney PJ: Experimental and computational approaches to estimate solubility and permeability in drug discovery and development settings. Adv Drug Deliv Rev 200I, 46(I-3):3-26.

4. Leeson PD and Davis AM: Time-related differences in the physical property profiles of oral drugs. J Med Chem 2004, 47 (25):6338-6348.

5. Frimurer TM, Bywater R, Naerum L, Lauritsen LN and Brunak S: Improving the odds in discriminating "drug-like" from "non drug-like" compounds. J Chem Inf Comput Sci 2000, 40 (6): $1315-1324$.

6. Ajay A, Walters WP and Murcko MA: Can we learn to distinguish between "drug-like" and "nondrug-like" molecules?. J Med Chem 1998, 4I(18):3314-3324.

7. Sadowski J and Kubinyi $\mathrm{H}$ : A scoring scheme for discriminating between drugs and nondrugs. J Med Chem 1998, 4I(18): 3325-3329.

8. Brustle M, Beck B, Schindler T, King W, Mitchell T and Clark T: Descriptors, physical properties, and drug-likeness. J Med Chem 2002, 45(16):3345-3355.

9. Oprea TI: Property distribution of drug-related chemical databases. J Comput Aided Mol Des 1999, 14:25I-264.

10. Congreve $M$, Carr R, Murray $C$ and Jhoti H: A 'rule of three' for fragment-based lead discovery?. Drug Discov Today 2003, 8(19): 876-877.

1I. Ertl P, Roggo S and Schuffenhauer A: Natural product-likeness score and its application for prioritization of compound libraries. J Chem Inf Model 2008, 48(I):68-74.

12. Gupta $S$ and Aires-de-Sousa J: Comparing the chemical spaces of metabolites and available chemicals: models of metabolite-likeness. Mol Divers 2007, I I(I):23-36.

13. Eckert $\mathrm{H}$ and Bajorath J: Exploring peptide-likeness of active molecules using 2D fingerprint methods. J Chem Inf Model 2007, 47(4): $1366-1378$.

14. Oprea T: Cheminformatics and the quest for leads in drug discovery. Handbook of Chemoinformatics Wiley-VCH: J Gasteiger, Weinheim 2003, I508-153I.

15. Oprea TI: Current trends in lead discovery: are we looking for the appropriate properties?. J Comput Aided Mol Des 2002, 16(5-6):325-334.

16. Oprea TI, Davis AM, Teague SJ and Leeson PD: Is there a difference between leads and drugs? A historical perspective. J Chem Inf Comput Sci 200I, 4I(5): I308-13I5.

17. Jorissen RN and Gilson MK: Virtual screening of molecular databases using a support vector machine. J Chem Inf Model 2005, 45(3):549-561.

18. Henkel T, Brunne RM, Muller $\mathrm{H}$ and Reichel F: Statistical investigation into the structural complementarity of natural products and synthetic compounds. Angewandte Chemie-International Edition 1999, 38(5):643-647.

19. Stahura FL, Godden JW, Xue L and Bajorath J: Distinguishing between natural products and synthetic molecules by descriptor Shannon entropy analysis and binary QSAR calculations. J Chem Inf Comput Sci 2000, 40(5): 1245-1252.

20. Feher $M$ and Schmidt JM: Property distributions: differences between drugs, natural products, and molecules from combinatorial chemistry. J Chem Inf Comput Sci 2003, 43 (I):2।8-227.

21. Lee ML and Schneider G: Scaffold architecture and pharmacophoric properties of natural products and trade drugs: application in the design of natural product-based combinatorial libraries. J Comb Chem 2001, 3(3):284-289.

22. Hattori M, Okuno $Y$, Goto $S$ and Kanehisa M: Development of a chemical structure comparison method for integrated analysis of chemical and genomic information in the metabolic pathways. J Am Chem Soc 2003, I 25(39): | I 853-| | 865. 
23. Nobeli I, Ponstingl H, Krissinel EB and Thornton JM: A structurebased anatomy of the E. coli metabolome. J Mol Biol 2003, 334 (4):697-719.

24. Karakoc E, Sahinalp SC and Cherkasov A: Comparative QSARand fragments distribution analysis of drugs, druglikes, metabolic substances, and antimicrobial compounds. $J$ Chem Inf Model 2006, 46(5):2167-2I82.

25. Dobson PD, Patel $Y$ and Kell DB: 'Metabolite-likeness' as a criterion in the design and selection of pharmaceutical drug libraries. Drug Discov Today 2009, | 4( I-2):3 |-40.

26. Gleeson MP: Generation of a set of simple, interpretable ADMET rules of thumb. I Med Chem 2008, 5 I (4):8I7-834

27. Schuster $D$, Laggner $C$ and Langer $T$ : Why drugs fail-a study on side effects in new chemical entities. Curr Pharm Des 2005, I I (27):3545-3559.

28. Gut J and Bagatto D: Theragenomic knowledge management for individualised safety of drugs, chemicals, pollutants and dietary ingredients. Expert Opin Drug Metab Toxicol 2005, I (3):537-554.

29. Hansch C, Bjorkroth JP and Leo A: Hydrophobicity and central nervous system agents: on the principle of minimal hydrophobicity in drug design. J Pharm Sci 1987, 76(9):663-687.

30. Veber DF, Johnson SR, Cheng HY, Smith BR, Ward KW and Kopple KD: Molecular properties that influence the oral bioavailability of drug candidates. I Med Chem 2002, 45 (I 2):2615-2623

31. Wishart DS, Tzur D, Knox C, Eisner R, Guo AC, Young N, Cheng D, Jewell K, Arndt D and Sawhney S, et al: HMDB: the Human Metabolome Database. Nucleic Acids Res 2007, 35 Database: D521-526.

32. Wishart DS, Knox C, Guo AC, Shrivastava S, Hassanali M, Stothard P, Chang Z and Woolsey J: DrugBank: a comprehensive resource for in silico drug discovery and exploration. Nucleic Acids Res 2006, 34 Database: D668-672.

33. Shin JM and Cho DH: PDB-Ligand: a ligand database based on PDB for the automated and customized classification of ligand-binding structures. Nucleic Acids Res 2005, 33 Database: D238-241.

34. Gold LS, Sawyer CB, Magaw R, Backman GM, de Veciana M, Levinson R, Hooper NK, Havender WR, Bernstein L and Peto R, et al: A carcinogenic potency database of the standardized results of animal bioassays. Environ Health Perspect 1984 58:9-319.

35. SciTegic Pipeline Pilot. Accelrys, Inc., San Diego, CA, USA; http://accelrys.com/products/scitegicl.

36. Zhou D, Alelyunas $Y$ and Liu R: Scores of extended connectivity fingerprint as descriptors in QSPR study of melting point and aqueous solubility. I Chem Inf Model 2008, 48(5):981-987.

37. Hert J, Willett P, Wilton DJ, Acklin P, Azzaoui K, Jacoby E and Schuffenhauer A: Comparison of topological descriptors for similarity-based virtual screening using multiple bioactive reference structures. Org Biomol Chem 2004, 2(22):3256-3266.

38. Ghose $A K$ and Crippen GM: Atomic physicochemical parameters for three-dimensional-structure-directed quantitative structure-activity relationships. 2. Modeling dispersive and hydrophobic interactions. J Chem Inf Comput Sci 1987, 27(I): $2 \mathrm{I}-35$.
Publish with BioMed Central and every scientist can read your work free of charge

"BioMed Central will be the most significant development for disseminating the results of biomedical research in our lifetime. "

Sir Paul Nurse, Cancer Research UK

Your research papers will be:

- available free of charge to the entire biomedical community

- peer reviewed and published immediately upon acceptance

- cited in PubMed and archived on PubMed Central

- yours - you keep the copyright
BioMedcentral 